\title{
The role of pacing rate in the modulation of mechano-induced immediate and delayed changes in the force and Ca-transient of cardiac muscle
}

\author{
Oleg Lookin ${ }^{\mathrm{a}, \mathrm{b},{ }^{*}}$, Xenia Butova ${ }^{\mathrm{a}, \mathrm{b}}$, Yuri Protsenko ${ }^{\mathrm{a}}$ \\ a Institute of Immunology and Physiology, Ural Branch of Russian Academy of Sciences, 620049, 106 Pervomayskaya St., Yekaterinburg, Russia \\ ${ }^{\mathrm{b}}$ Center for Fundamental Biotechnology and Bioengineering, Institute of Natural Sciences and Mathematics, Ural Federal University, 620002,19 Mira St., \\ Yekaterinburg, Russia
}

\section{A R T I C L E I N F O}

\section{Article history:}

Received 16 October 2019

Received in revised form

11 March 2020

Accepted 11 May 2020

Available online 23 May 2020

\section{Keywords:}

Myocardium

Systolic shortening-re-lengthening

Mechano-calcium feedback

Calcium transient

(intra)cellular calcium balance

\begin{abstract}
A B S T R A C T
Myocardial function is tuned by dynamic changes in length and load via mechano-calcium feedback. This regulation may be significantly affected by heart rhythm. We evaluated the mechano-induced modulation of contractility and Ca-transient (CaT) in the rat myocardium subjected to twitch-by-twitch shortening-re-lengthening $(\downarrow-\uparrow)$ trains of different lengths $(N=1 \ldots 720$ cycles) at low $(1 \mathrm{~Hz})$ and near-physiological $(3.5 \mathrm{~Hz}$ ) pacing rates. Force/CaT characteristics were evaluated in the first post-train isometric twitch (immediate effect) and during slow changes (delayed maximal elevation/decrease) and compared with those of the pre-train twitch. The immediate inotropic effect was positive for $N=30$ $\ldots 720$ and negative for $N=1 \ldots 20$, while the delayed effect was always positive. The immediate and delayed inotropic effects were significantly higher at $3.5-\mathrm{Hz} v s \quad 1-\mathrm{Hz}(P<0.05)$. The prominent inotropism was accompanied by much smaller changes in the CaT diastolic level/amplitude. The shortening -re-lengthening train induced oscillations of the slow change in force at $3.5-\mathrm{Hz}$ (always) and at $1-\mathrm{Hz}$ ( $50 \%$ of muscles), which were dependent of the train length and independent of the pacing rate. We suggest that twitch-by-twitch shortening-re-lengthening of cardiac muscle decreases $\mathrm{Ca}^{2+}$ buffering by troponin $\mathrm{C}$ and elevates $\mathrm{Ca}^{2+}$ loading of the sarcoplasmic reticulum (SR); the latter cumulatively depends on the train length. A high pacing rate intensifies the cumulative transient shift in the $\mathrm{SR} \mathrm{Ca}^{2+}$ loading, augmenting the post-train inotropic response and prolonging its recovery to the pre-train level. The pacing-dependent mechano-induced inotropic effects remain to be elucidated in the myocardium with impaired Ca handling.
\end{abstract}

(c) 2020 Elsevier Ltd. All rights reserved.

\section{Introduction}

In the body, the myocardium constantly adapts its contractile function to the dynamic mechanical conditions and pacing rate, providing concerted and powerful contractions of the whole heart (Endoh, 2004; Ferreira-Martins and Leite-Moreira, 2010; ter Keurs, 2012; Milani-Nejad et al., 2014; Biesiadecki et al., 2014; Paterek et al., 2016). The inherent mechanisms of this adaptation involve the kinetics of free cytosolic calcium (Ca-transient), which regulates the interaction of myofilaments (Hanft et al., 2008; ter Keurs, 2012; Eisner et al., 2017). Following the rapid elevation of free cytosolic $\mathrm{Ca}^{2+}$ during activation of the cell, the sarcoplasmic

\footnotetext{
* Corresponding author. 620049, 106 Pervomayskaya st., Yekaterinburg, Russia. E-mail address: o.lookin@iip.uran.ru (O. Lookin).
}

reticulum (SR) and regulatory protein troponin $\mathrm{C}(\mathrm{TnC})$ of thin filaments both begin to communicate with $\mathrm{Ca}^{2+}$ ions. The amount of $\mathrm{Ca}^{2+}$ loading of the SR during the current beat is determined both by the SERCA2a pumping and $\mathrm{Ca}^{2+}$ leak from the SR, and the level of cytosolic $\mathrm{Ca}^{2+}$ affects these oppositely directed $\mathrm{Ca}^{2+}$ fluxes (Eisner et al., 2000, 2017; Trafford et al., 2001; Smith and Eisner, 2019), whereas the concurrent $\mathrm{Ca}^{2+}$ buffering by $\mathrm{TnC}$ is highly dependent on the mechanical conditions, that is, dynamical changes in length and load (Dobesh et al., 2002; de Tombe et al., 2010; ter Keurs, 2012; Korte et al., 2012). The interplay between SERCA2a (which is a relatively slow and mechano-independent $\mathrm{Ca}^{2+}$-buffer) and $\mathrm{TnC}$ (a relatively fast and mechano-dependent $\mathrm{Ca}^{2+}$-buffer) strongly determines the kinetics of the Ca-transient and provides normal Ca handling (Hanft et al., 2008; Briston et al., 2014). It is of great physiological importance that the 
mechano-dependent effects on Ca-TnC interaction in the preceding beats may greatly modulate $\mathrm{Ca}^{2+}$ homeostasis in cardiac cells, and consequently, influence their contractile response in the succeeding beats.

The heart rate limits the diastolic interval, which is important for the complete recovery of $\mathrm{Ca}^{2+}$ balance in current beat (Louch et al., 2012; Gattoni et al., 2016). Too high a pacing rate may result in the incomplete $\mathrm{Ca}^{2+}$ loading of the SR. Therefore, systolic shortening and re-lengthening via mechano-calcium feedback may substantially affect the $\mathrm{Ca}^{2+}$ balance at a high pacing rate compared with a low one. In turn, the transient mechano-induced shift in $\mathrm{Ca}^{2+}$ redistribution between different (intra)cellular $\mathrm{Ca}^{2+}$ sources may enhance, or alternatively, attenuate the contractile function of cardiac muscle.

In this study, we evaluated the immediate and delayed effects of twitch-by-twitch shortening-re-lengthening of the isolated rat trabecula on its force and Ca-transient under low and nearphysiological pacing rates. The influence of the pacing rate on the existence and expression of the observed effects was comprehensively assessed, and the role of the diastolic interval in the proper regulation of (intra)cellular $\mathrm{Ca}^{2+}$ fluxes was specifically addressed.

\section{Materials and methods}

The animals involved in the present study were cared according to the Guide for the Care and Use of Laboratory Animals published by the US National Institutes of Health (NIH Publication No. 85-23, revised 1985), their use was approved by the local Institutional Ethics Committee. Adult Wistar rats $(N=12)$, aged $2-3$ months, were obtained from the Institutional Animal House. They were maintained under standard conditions before use in the experiments.

\subsection{Muscle preparations}

Each animal was euthanised by rapid cervical dislocation immediately before the experiment. The heart was removed immediately following euthanasia and placed in a modified KrebsHenseleit buffer (KHB, in mM: $\mathrm{NaCl} 118, \mathrm{KCl} 4.7, \mathrm{MgSO}_{4} 1.2, \mathrm{KH}_{2} \mathrm{PO}_{4}$ 1.2, $\mathrm{NaHCO}_{3} 25$, HEPES $10, \mathrm{CaCl}_{2} 2$, glucose 11.1, pH adjusted to 7.35 at room temperature under continuous aeration with $95 \% \mathrm{O}_{2} /$ $5 \% \mathrm{CO}_{2}$ ) containing $30 \mathrm{mM}$ 2,3-butanedione monoxime (BDM). A thin trabecula (100-300 $\mu \mathrm{m}$ thick, $1-3 \mathrm{~mm}$ long) was dissected from the right ventricle and mechanically clipped to the levers of the force transducer and length servomotor in the experimental chamber perfused by BDM-free KHB under continuous aeration with $95 \% \mathrm{O}_{2} / 5 \% \mathrm{CO}_{2}$. The muscle was stimulated by rectangular electrical impulses of $\sim 20 \%$ above the threshold value. To monitor free cytosolic $\mathrm{Ca}^{2+}$, the muscles were preincubated in the saline containing $\mathrm{Ca}^{2+}$-sensitive fluorescent indicator (5 $\mu \mathrm{M}$ fura-2/ $\mathrm{AM}+0.4 \% \mathrm{w} / \mathrm{v}$ Pluronic F-127) for $\sim 1 \mathrm{~h}$ at room temperature and a $0.2-\mathrm{Hz}$ pacing rate. Prior to the measurements, the perfusion was continued in dye-free saline for $30 \mathrm{~min}$. The total number of muscle strips subjected to mechanical testing was $n=12$. All chemicals were purchased from Sigma Aldrich (USA).

\subsection{Data acquisition}

The mechanical response of the muscle was measured by the precise force transducer, and the muscle length was both monitored and dynamically applied by length servomotor (Muscle Research System; Scientific Instruments, Heidelberg, Germany). Catransients were recorded simultaneously with the mechanical response. The devices were synchronised with a personal computer using a DAC/ADC (PCI-1716S; AdLink Technology, New Taipei City,
Taiwan). Data were sampled at $10 \mathrm{kHz}$ using custom-made software running in a real-time environment (HyperKernel; Nematron, Ann Arbor, MI, USA) integrated into Windows XP (Microsoft; Redmond, WA, USA); they were then processed off-line using custom-made software (EqapAll6). Ca-transients were obtained as a ratio of emitting fluorescence collected at $510 \mathrm{~nm}$ by excitation at either $340 \mathrm{~nm}$ or $380 \mathrm{~nm}$ and presented here in a non-calibrated (raw) manner.

\subsection{The mechanical tests}

Since the muscle was equilibrated for at least $30 \mathrm{~min}$ at a $1-\mathrm{Hz}$ pacing rate and $25^{\circ} \mathrm{C}$, the isometric force-length protocol was implemented to determine the optimal muscle length ( $\left.L_{M A X}\right)$. Further mechanical tests were done at a preload of $95 \% L_{M A X}$. Each test comprised the application of a single half-sine shortening-relengthening $(\downarrow-\uparrow)$ cycle to the actively contracting muscle, that is, a single cycle per twitch, to imitate the systolic ejection phase typical of the ventricular chamber. To standardise the conditions between different preparations, the amplitude of the $\downarrow-\uparrow$ cycle was set to $20 \% L_{\text {MAX }}$. To reproduce the typical ejection cycle of the ventricle, the $\downarrow-\uparrow$ cycle was started and ended at the level of halfamplitude of isometric force at its rising and declining phases, respectively. The trains of twitch-by-twitch $\downarrow-\uparrow$ cycles with fixed numbers $(N=1,5,10,30,60,120,360$ and 720 , referred to throughout as the train length) were tested. Note that the actual duration of the train depends on both the train length and duration of a single twitch (inverse pacing rate). The typical design of a mechanical test is shown in Fig. 1A.

Immediate and delayed effects of the train of $\downarrow-\uparrow$ cycles on specific characteristics of isometric twitches and Ca-transients were calculated as a percentage of those characteristics measured in the pre-train isometric twitches (Fig. 1B). For example, the immediate effect was calculated as a percentage of the ratio between the values in the first post-train isometric twitch $\left(Y_{\text {after }}\right)$ and the steady-state pre-train isometric twitch ( $\left.Y_{\text {before }}\right)$, that is, Effect $=100 \% * Y_{\text {after }} / Y_{\text {before }}$. A value of Effect $=100 \%$ indicated no immediate changes in the characteristics. An Effect less or greater than $100 \%$ indicates lowering or elevation, respectively, of the characteristics compared with the pre-train state. To reveal the delayed effects of the train of $\downarrow-\uparrow$ cycles on the contractility and Ca-transient, we carefully examined post-train slow changes to find the twitches with highest and lowest values; we refer to them as the maximal elevation and maximal decrease, respectively ( $Y_{\max }$ and $Y_{\min }$ in Fig. 1B). The following characteristics of isometric twitches and Ca-transients were analysed in terms of their sensitivity to the $\downarrow-\uparrow$ cycles, using the following parameters: diastolic level, amplitude, time-to-peak and time to decline from peak to $50 \%$ amplitude $\left(T_{50}\right)$. The stability of contractions/Ca-transients of the muscle was confirmed before the analysis (see Supplemental data, Fig. S3).

In our preliminary tests of the effects of $\downarrow-\uparrow$ cycles on the contractility of the cardiac trabecula, we found a minor contribution of the preload $\left(85-100 \% L_{M A X}\right)$, temperature $\left(25^{\circ} \mathrm{C}, 30^{\circ} \mathrm{C}, 38^{\mathrm{O}} \mathrm{C}\right)$ and duration of the $\downarrow-\uparrow$ cycle (50-300 ms) on the extent of these effects (see Supplemental data for details). As expected, the effect of the $\downarrow-\uparrow$ cycles was directly proportional to the amplitude of shortening; however, the train length had even greater effect. In addition, the extent of the observed effects was strikingly dependent on the pacing rate (see Supplemental data for details). At a near-physiological pacing rate, the effects were sizeable compared with those at a low pacing rate. The effect of saline temperature was found to be minor compared with the pacing rate. Because of the fast exhaustion of the metabolic reserve of cardiac muscle and due to the substantial degeneration of Ca-sensitive fluorescent dye 

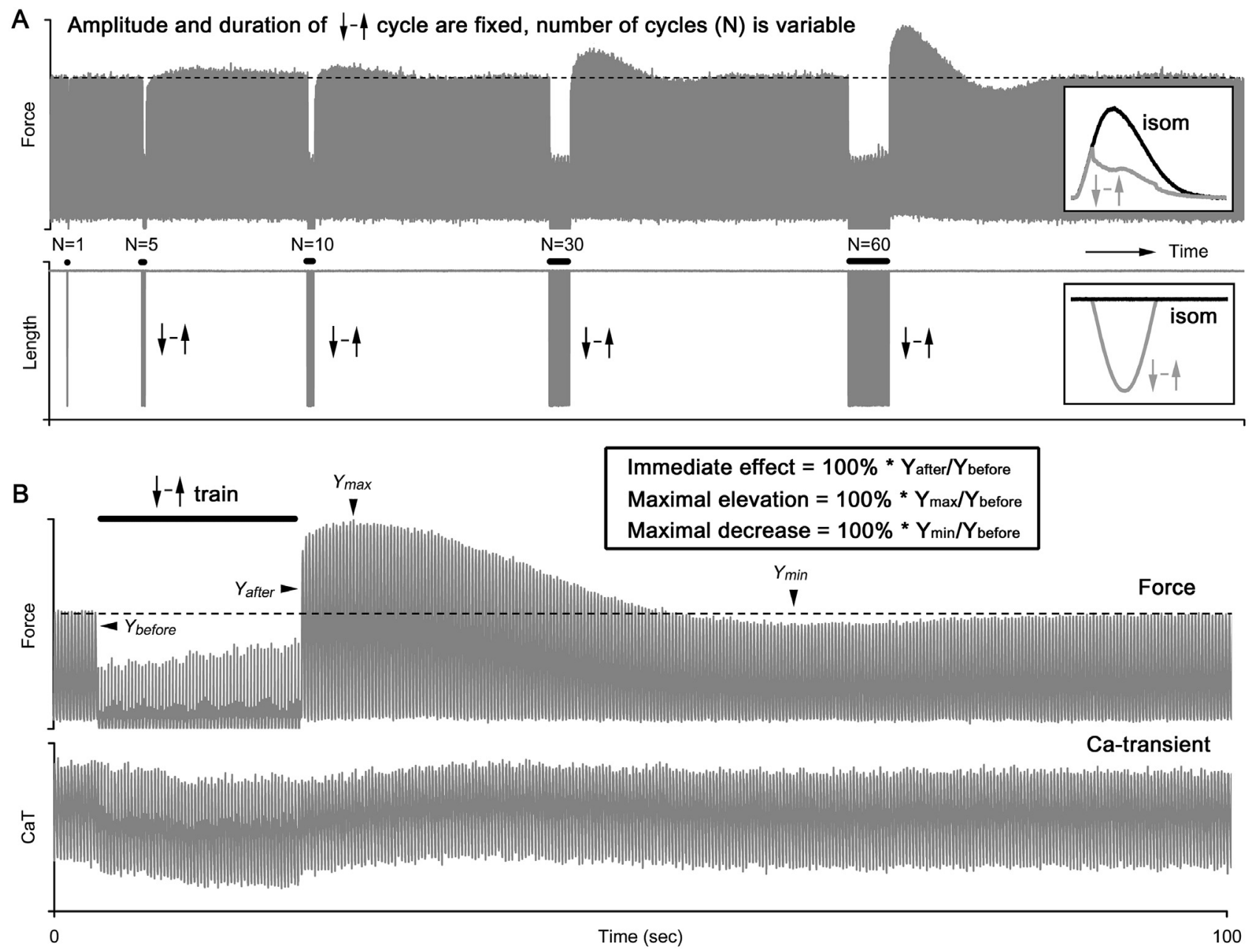

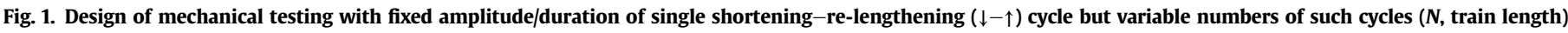

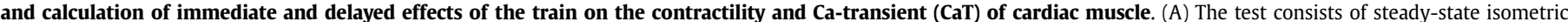

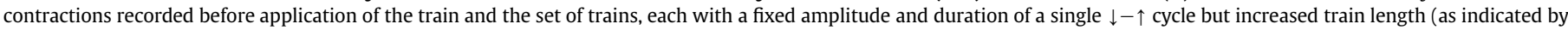

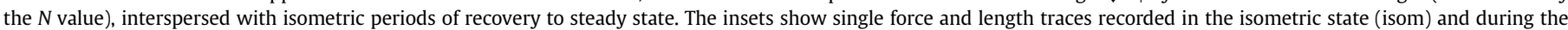

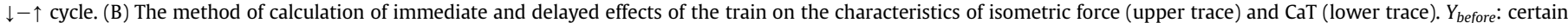

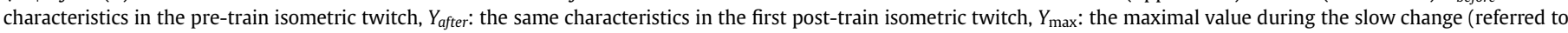

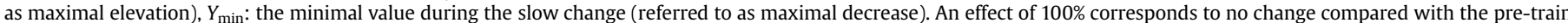

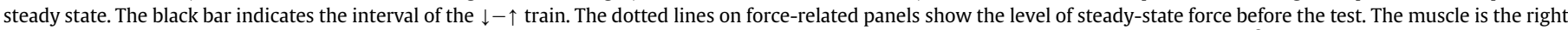
ventricular trabecula, preload is $95 \% L_{M A X}$, amplitude of shortening is $20 \% L_{M A X}$, pacing rate is $1 \mathrm{~Hz}(\mathrm{~A})$ and $3.5 \mathrm{~Hz}(\mathrm{~B})$, and temperature is $25^{\circ} \mathrm{C}$.

fura-2 at a physiological temperature, we conducted our tests under substantially sub-physiological temperature $\left(25^{\circ} \mathrm{C}\right)$ at nonphysiological $(1 \mathrm{~Hz})$ and near-physiological pacing rates $(3.5 \mathrm{~Hz})$.

\subsection{Statistical analysis}

The Mann-Whitney $U$ test was used to evaluate the significance of the difference between the mean values of the immediate effect, maximal elevation or maximal decrease of certain characteristics of isometric force and Ca-transient obtained for the same train length at the low $(1-\mathrm{Hz})$ and near-physiological $(3.5-\mathrm{Hz})$ pacing rates. Oneway repeated measures analysis of variance (ANOVA) was used to evaluate whether the increase in pacing rate significantly elevated the effect on individual muscle at the given train length. The differences were considered significant at $P<0.05$. Data are presented as the mean \pm standard deviation (S.D.).

\section{Results}

\subsection{The effect of the $\downarrow-\uparrow$ train on the contractile response}

The immediate effect of the train of $\downarrow-\uparrow$ cycles was strongly dependent on the train length ( $N$ value) at both the $1-\mathrm{Hz}$ and $3.5-\mathrm{Hz}$ pacing rates (Fig. 2A). On the other hand, starting from a certain train length, the effect did not elevate further, displaying 'saturation'. This occurred at $N=120$ or higher for the $1-\mathrm{Hz}$ pacing rate and at $N=360$ or higher for $3.5-\mathrm{Hz}$ pacing rate. Importantly, the effect was $>100 \%$ (i.e. positive inotropy) at a high enough train length and was found to be several times higher under the $3.5-\mathrm{Hz}$ pacing rate compared with the $1-\mathrm{Hz}$ rate; for example, at $N=720$, it was $121.4 \pm 32.4 \%$ at the $1-\mathrm{Hz}$ vs $211.3 \pm 87.1 \%$ at the $3.5-\mathrm{Hz}$ $(P<0.05)$. In contrast, short trains $(N<20)$ induced inactivation of the first post-train isometric twitch at both pacing rates (see inset in Fig. 2D). Surprisingly, at the $3.5-\mathrm{Hz}$ pacing rate, this inactivating effect was even higher, reaching $~ 90 \%$ of the pre-train value 
A

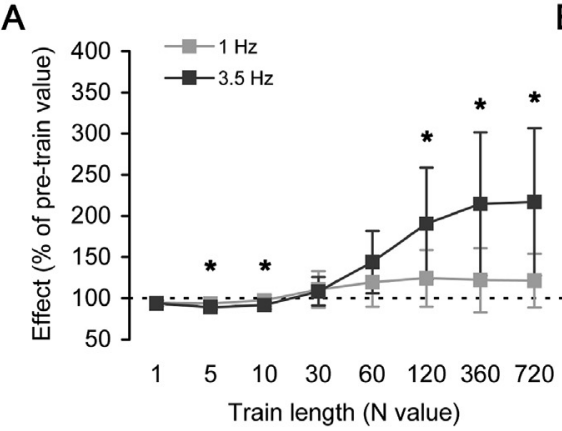

B

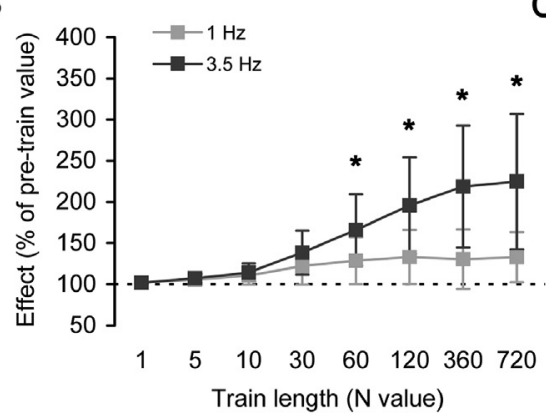

C

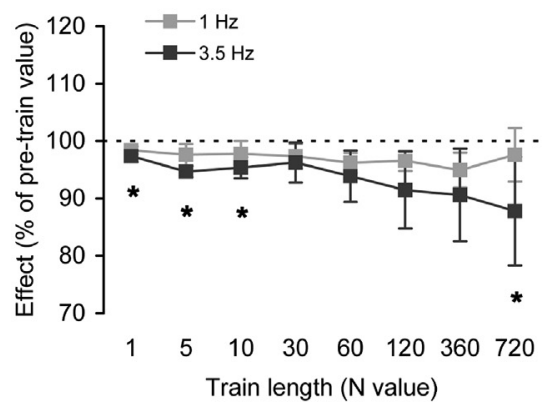

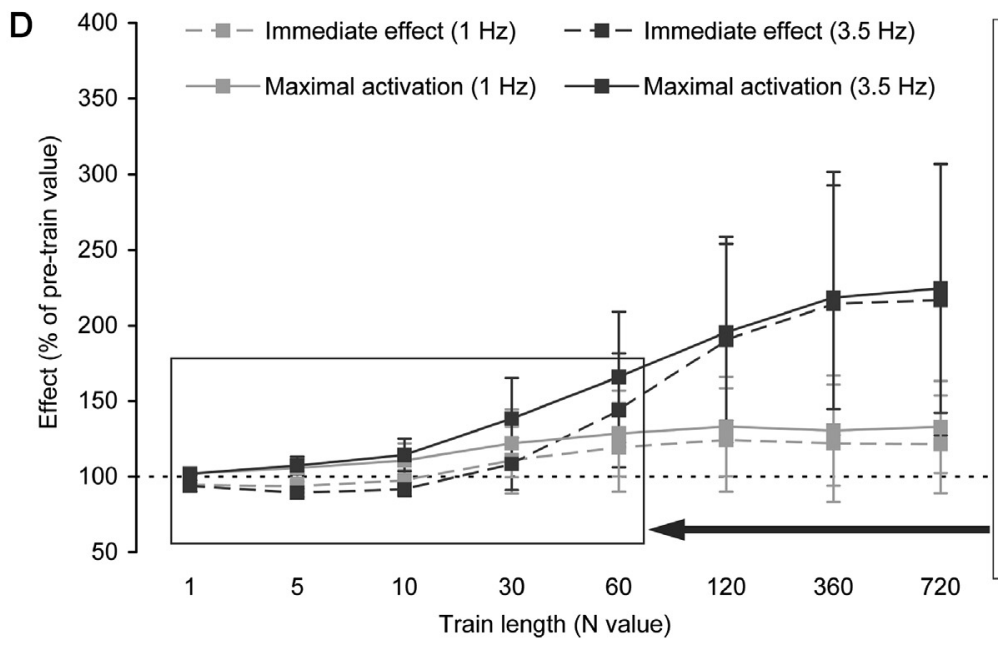

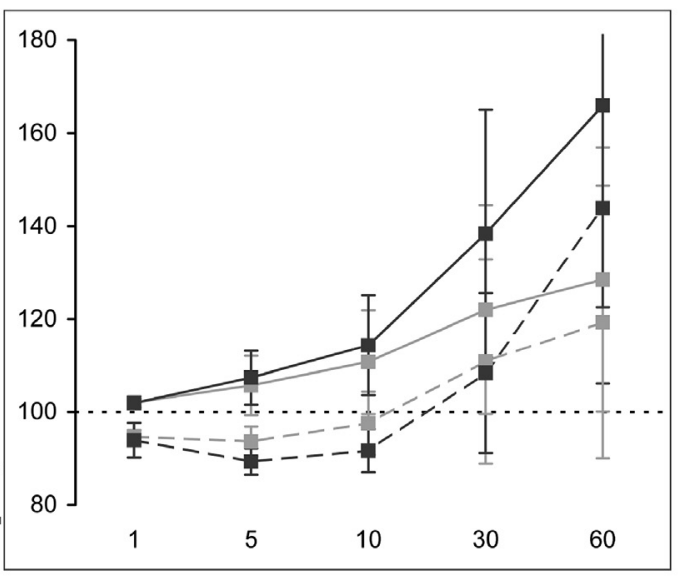

2. Effect of train length ( $\boldsymbol{N}$ value) on immediate and delayed changes in peak isometric force. Amplitude of isometric force in the first post-train twitch (A), maximal

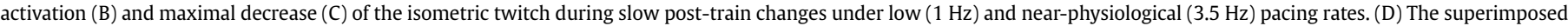

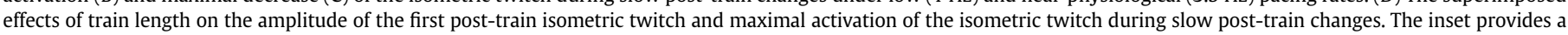

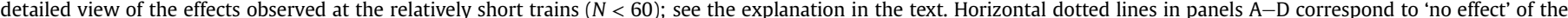

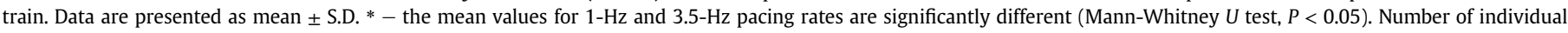
muscles $=10$.

(negative inotropy). Moreover, the relationship between the effect and train length was non-monotonic for short trains; that is, the inactivation of the first post-train isometric twitch was smaller at $N=1$ but higher at $N \approx 5$. With a further increase of $N$, the inactivation decreased again until no effect was seen at $N \approx 15 \ldots 20$.

The maximal elevation of peak force during slow changes rose with train length until a certain level, showing 'saturation' highly comparable to the immediate effects discussed above (Fig. 2B). This delayed effect was significantly higher at the $3.5-\mathrm{Hz}$ pacing rate for the trains of $N=60$ or higher $(P<0.05)$. In contrast, the maximal inactivation of force during slow changes was found to be dependent of the train length only at the $3.5-\mathrm{Hz}$ pacing rate, whereas minor effects were observed for the $1-\mathrm{Hz}$ pacing rate (Fig. $2 \mathrm{C}$ ). The timing characteristics of the slow changes in force also depended on the train length (see Section 3.3 and Fig. 9C below).

Fig. 2D superimposes the immediate and maximal activation effects as they relate to the train length. The inset in the figure provides a detailed view of the difference between immediate and maximal effects of the train on the peak isometric force. As can be seen, a short train inactivates the first post-train isometric twitch, which is nevertheless followed by the slow elevation in peak force over the pre-train value. A long train always potentiates both the first and subsequent twitches. Thus, whether the first post-train isometric twitch is inactivated by the train, the further slow posttrain changes always display elevation of the peak isometric force above the pre-train value at both the low and near-physiological pacing rates.

As evident from Fig. 2B, the maximal delayed effect of the $\downarrow-\uparrow$ train on peak isometric force is significantly higher at the $3.5-\mathrm{Hz}$ pacing rate compared with the $1-\mathrm{Hz}$ pacing rate, but only for $N \geq 60$ cycles. However, the precise analysis of the effect of shorter trains in each individual muscle revealed that, while the mean values are not significantly different for short trains, the effect is still enhanced at the $3.5-\mathrm{Hz}$ pacing rate (Fig. 3). This was observed in all muscles (except one) for $N=30$ cycles (Fig. 3, last panel in the top row), although the mean effects were not significantly different between the rates. The same was found for $N=10$ cycles, where 8 of 10 muscles showed a significantly enhanced effect at the $3.5-\mathrm{Hz}$ pacing rate (Fig. 3, third panel in the top row).

The effects of the $\downarrow-\uparrow$ train on the time-to-peak force and relaxation time to $50 \%\left(T_{50}\right)$ had a high resemblance to those found in the peak isometric force. At the $3.5-\mathrm{Hz}$ pacing rate, the increase in train length was accompanied by the substantial retardation of the first post-train isometric twitch (Fig. 4A and D); a much lower effect was found at the $1-\mathrm{Hz}$ pacing rate. Similarly, maximal elevation of time-to-peak and $T_{50}$ values during slow post-train changes reached $\sim 115-120 \%$ of the pre-train value at $3.5 \mathrm{~Hz}$, while this effect was much smaller at $1 \mathrm{~Hz}$ (Fig. 4B and E). In contrast, the maximal decrease in both time values did not depend on the pacing rate (Fig. $4 \mathrm{C}$ and F). In general, there was a slow change in both the amplitude and timing properties of isometric twitch after cyclic shortening-re-lengthening.

\subsection{The effect of the $\downarrow-\uparrow$ train on Ca-transient characteristics}

The immediate and delayed post-train slow changes in peak 

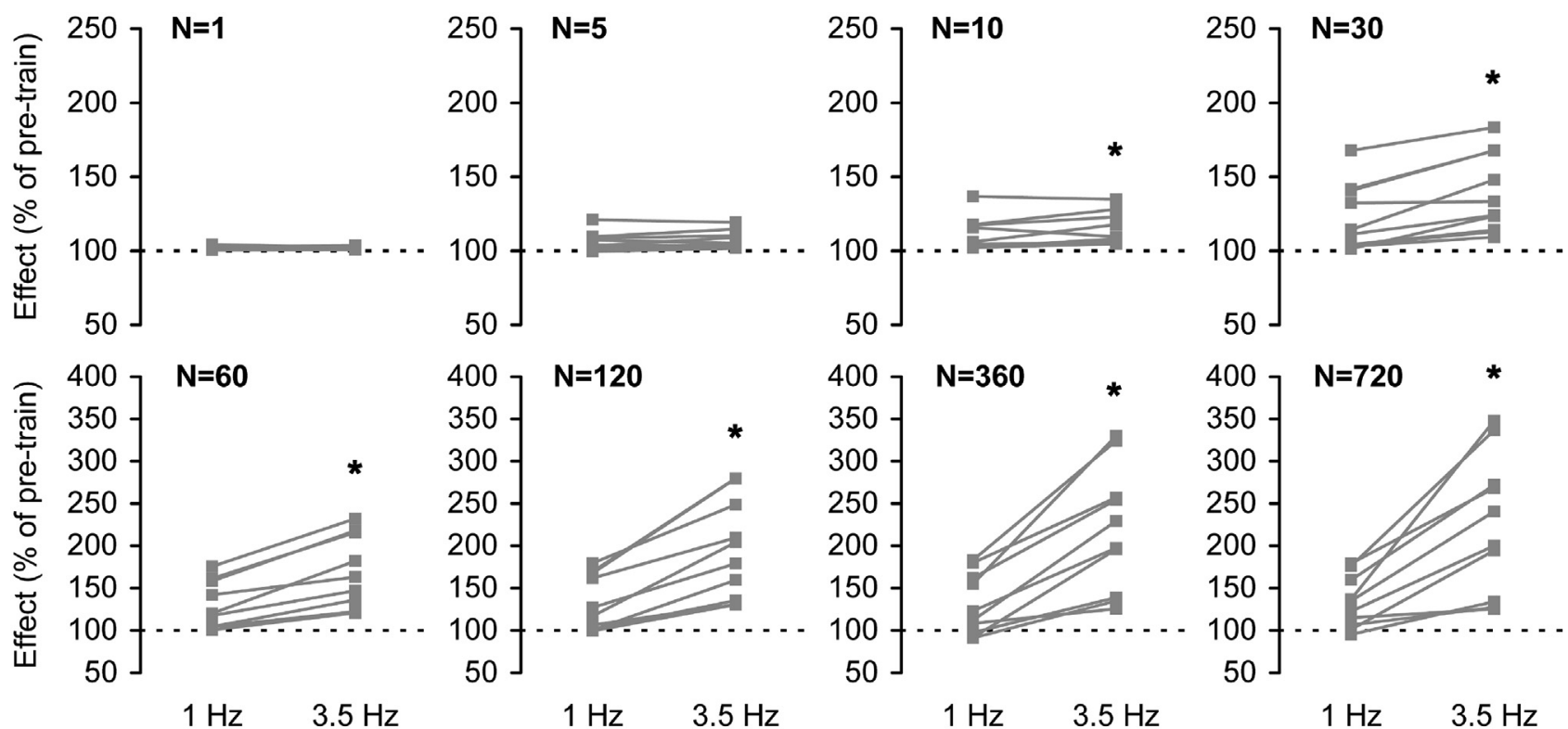

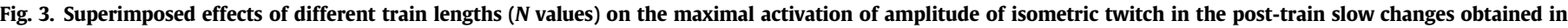

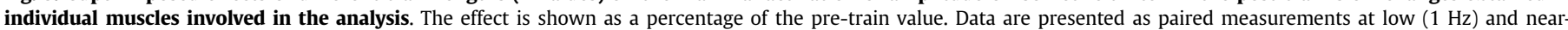

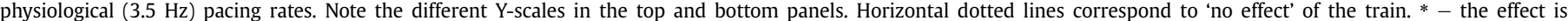
significantly higher at $3.5-\mathrm{Hz}$ vs $1-\mathrm{Hz}$ pacing rate in the muscle set (one-way paired measures ANOVA, $P<0.05$ ). Number of individual muscles $=10$.

A

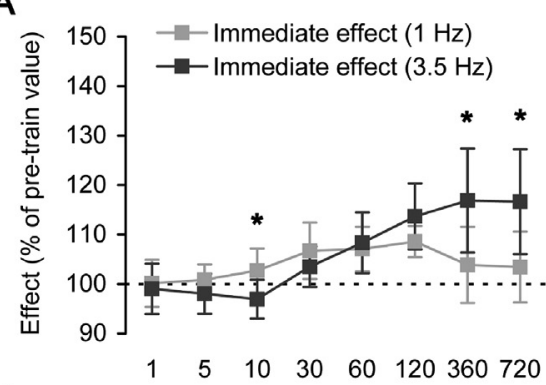

D

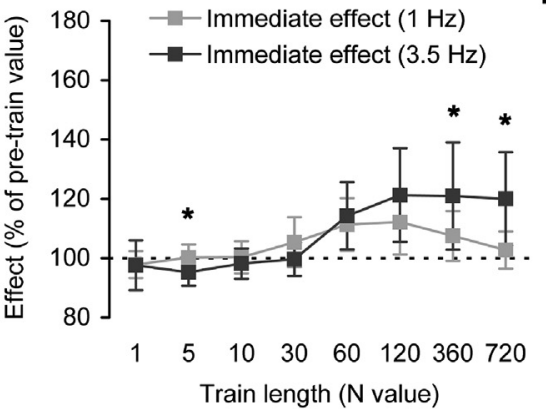

B

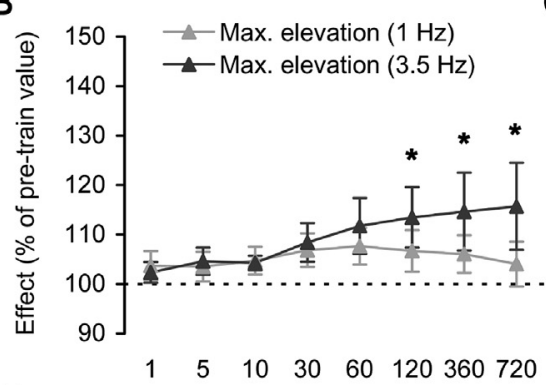

$\mathrm{E}$

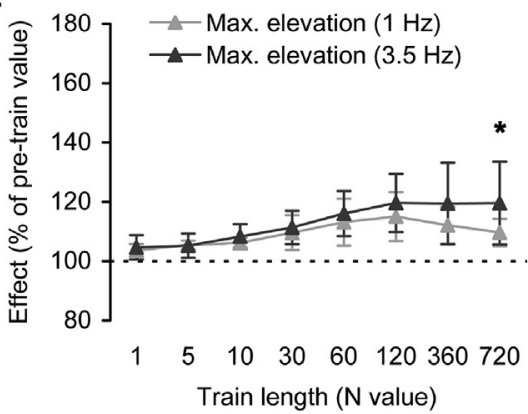

C

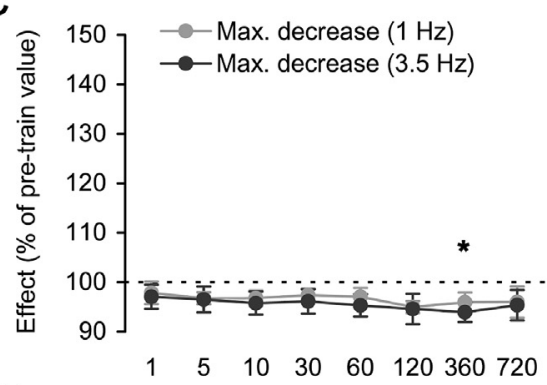

$\mathbf{F}$

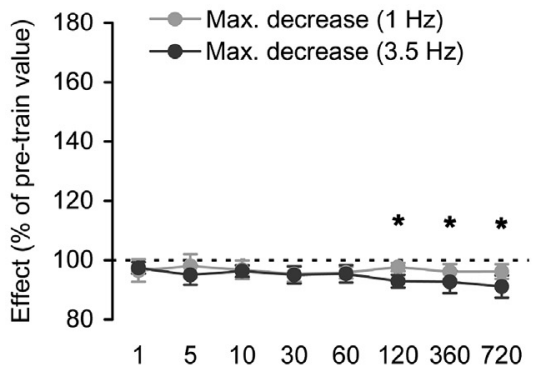

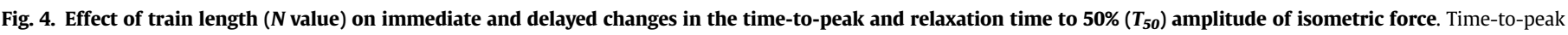

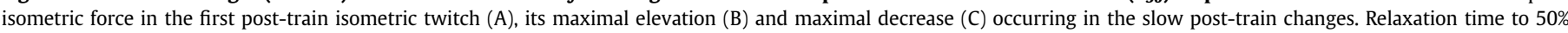

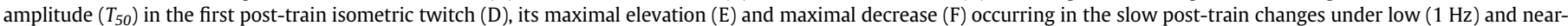

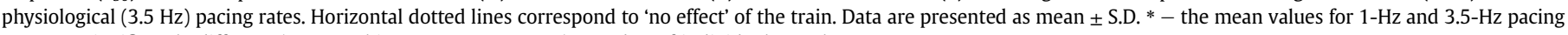
rates are significantly different (Mann-Whitney $U$ test, $P<0.05$ ). Number of individual muscles $=10$.

isometric force may originate from the corresponding changes in Ca-transient kinetics. We continuously monitored Ca-transient in the pre-train, during the train and in the post-train slow response (Fig. 5A). Note the twitch-to-twitch changes in the diastolic and peak systolic levels of Ca-transient for both in-train and post-train twitches, as well as the kinetics of Ca-transient decline (Fig. 5B). The longer the train, the greater the changes were observed. The representative record in Fig. 5A demonstrates that repetitive $\downarrow-\uparrow$ cycles induce a gradual decrease in the Ca-transient diastolic level in parallel with the gradual elevation of its amplitude. In the first post-train twitch, both substantially differed from their pre-train levels (Fig. 5B, last panel). However, the Ca-transient amplitude dropped back to the pre-train level during several twitches, whereas the Ca-transient diastolic level showed much slower 
A
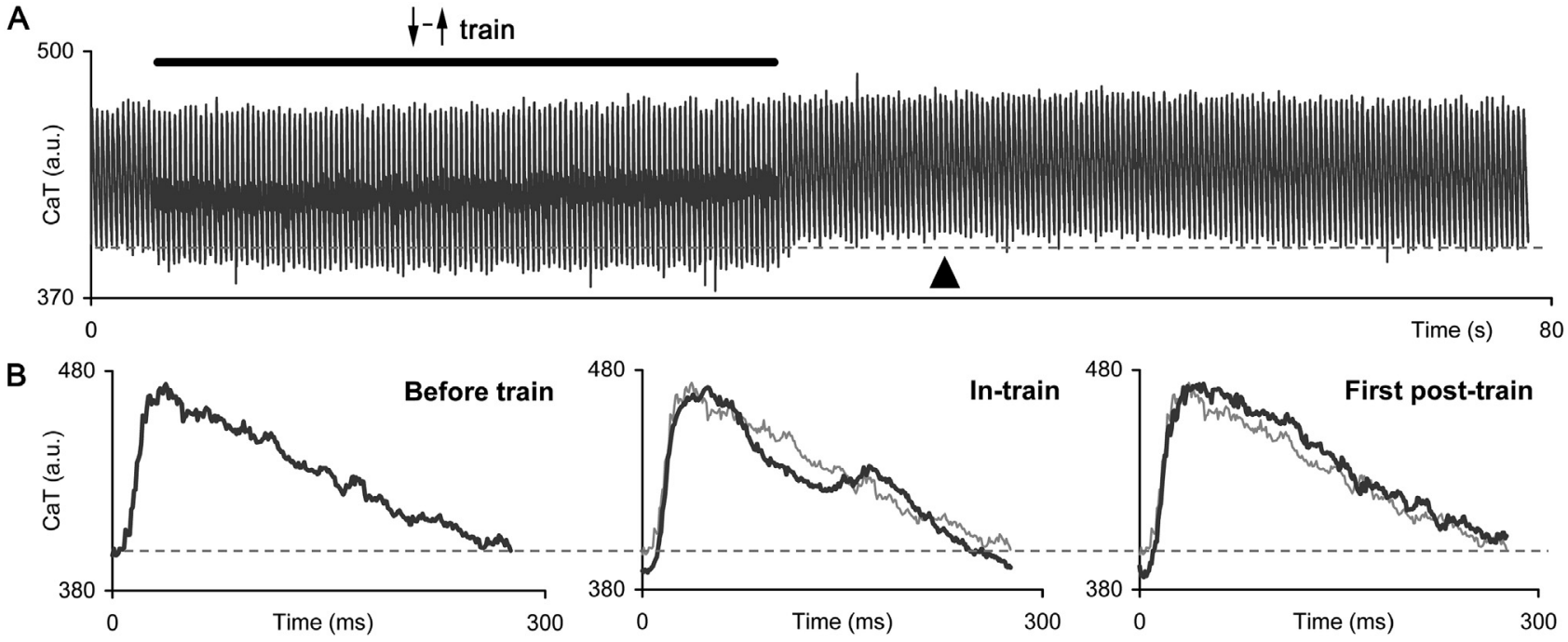

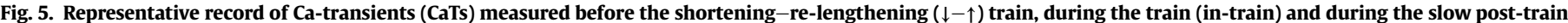

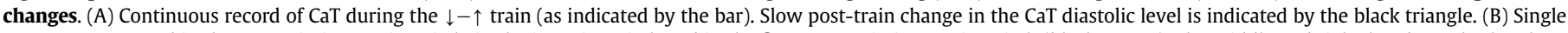

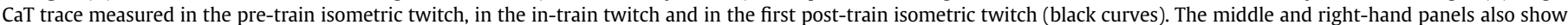

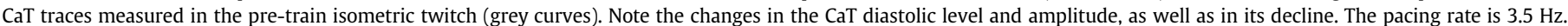

A

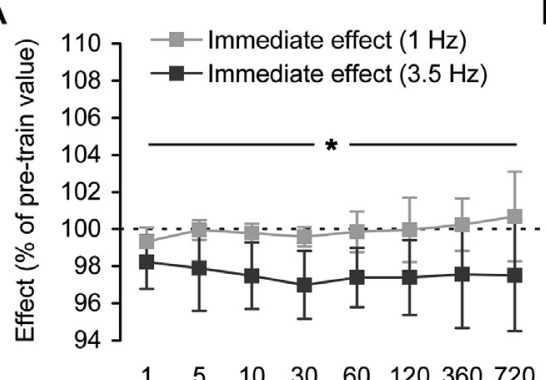

D

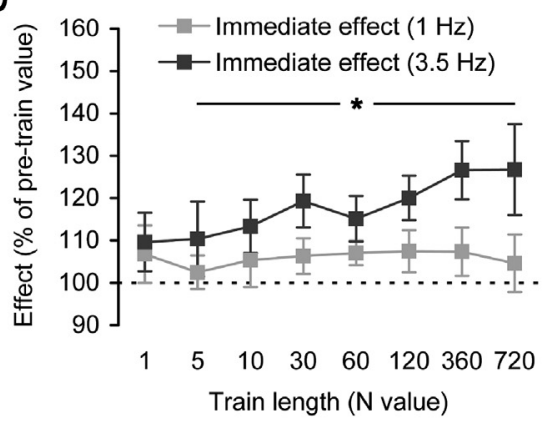

B

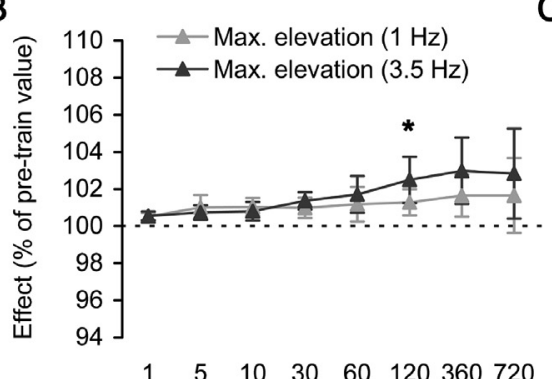

E

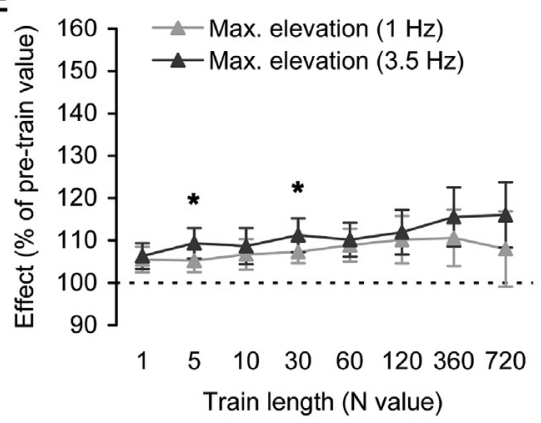

C

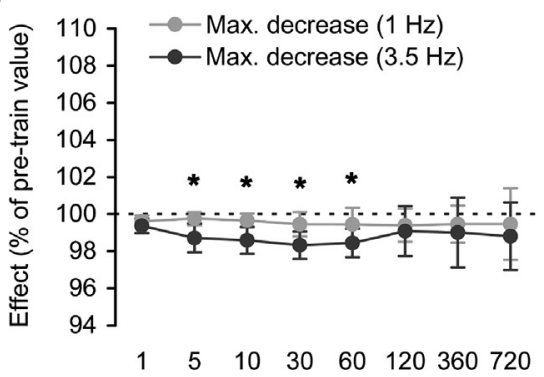

$\mathbf{F}$

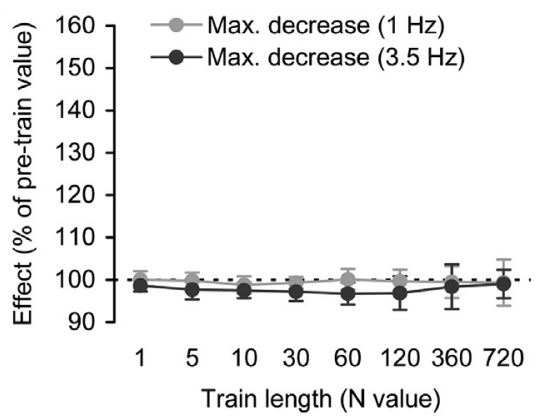

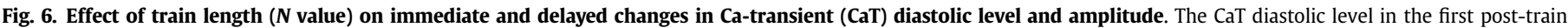

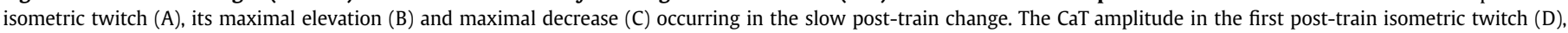

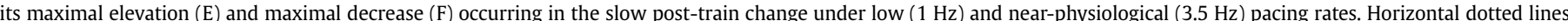

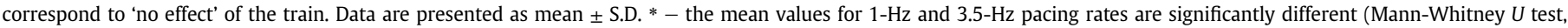
$P<0.05)$. Number of individual muscles $=10$.

changes, that is, an initial elevation above the pre-train level followed by a slow return to this level (as indicated by black triangle in Fig. 5A).

The immediate and delayed effects of the $\downarrow-\uparrow$ train on Catransient were minor at the $1-\mathrm{Hz}$ but not at the $3.5-\mathrm{Hz}$ pacing rate. The Ca-transient diastolic level in the first post-train isometric twitch was not affected by a train of any length at $1-\mathrm{Hz}$ pacing rate, but it was substantially lower than the pre-train level at $3.5-\mathrm{Hz}$ pacing rate (Fig. 6A). In addition, the maximal elevation of the Ca-transient diastolic level in the slow post-train changes amounted to several percentage points of the pre-train value at the 3.5-Hz pacing rate (Fig. 6B). In contrast to the diastolic level, the Catransient amplitude was always higher in the first post-train twitch $v s$ pre-train twitch, and this effect was observed at either pacing rate (Fig. 6D). Importantly, even the shortest train $(N=1)$ induced immediate elevation of the Ca-transient amplitude in the next isometric twitch; for example, if averaged for all muscles, these elevations were $6.8 \pm 6.8 \%$ and $9.6 \pm 6.9 \%$ over the pre-train value at $1-\mathrm{Hz}$ and $3.5-\mathrm{Hz}$ pacing rates, respectively (mean \pm S.D., no significant difference between the two means). Surprisingly, the 
A

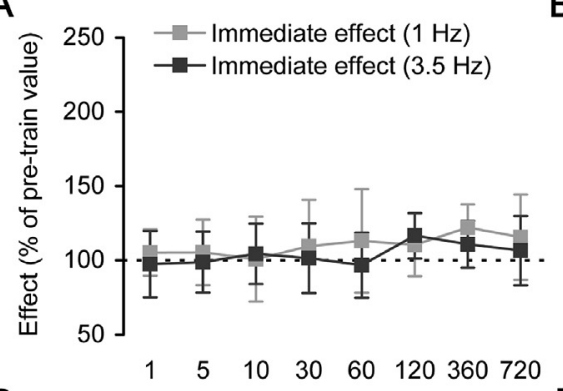

D

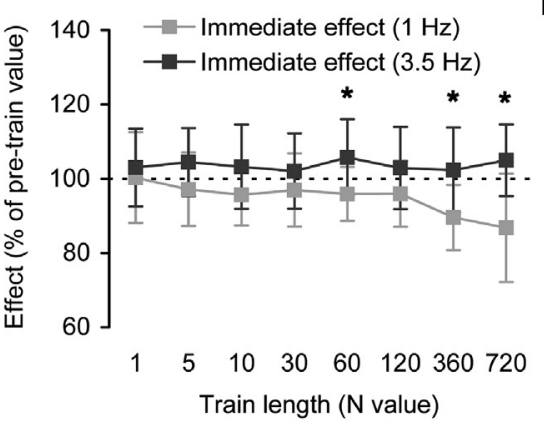

B

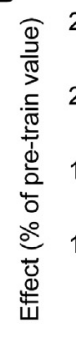

$\mathrm{E}$

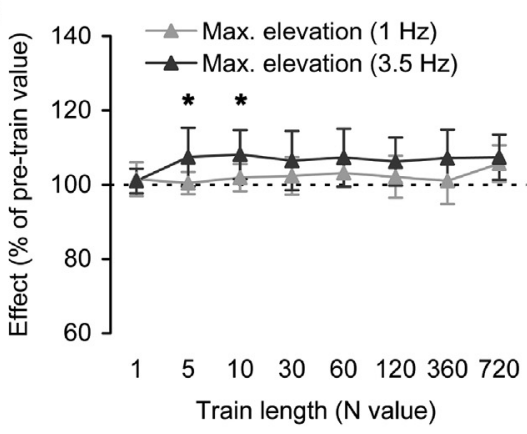

C

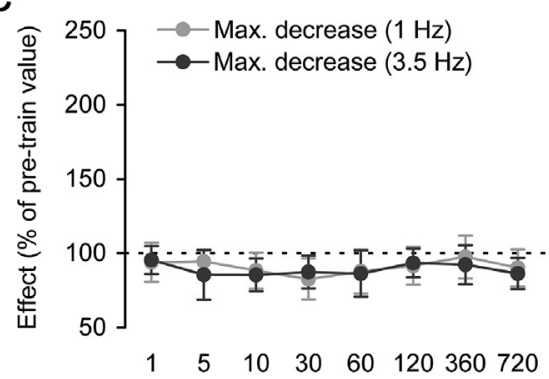

$F$

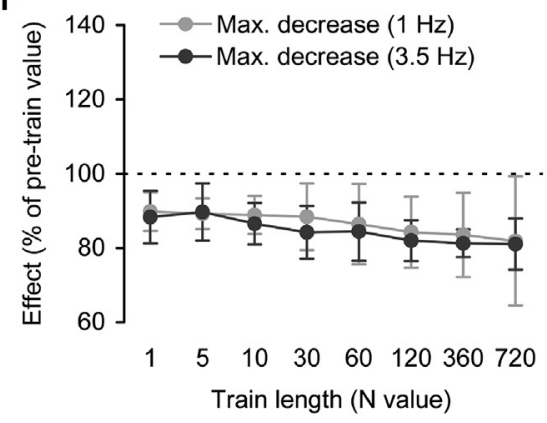

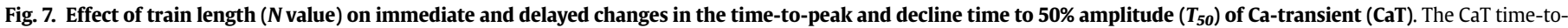

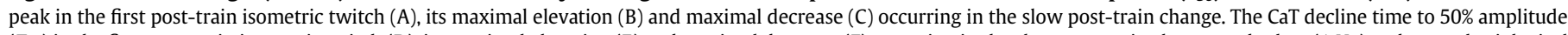

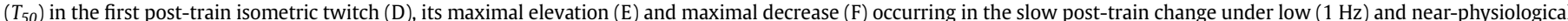

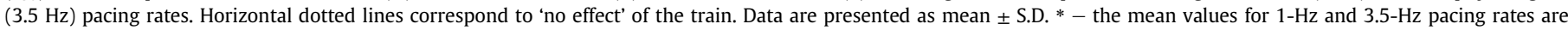
significantly different (Mann-Whitney $U$ test, $P<0.05$ ). Number of individual muscles $=10$.

A

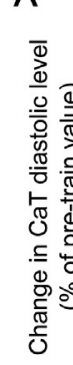

\section{$3.5 \mathrm{~Hz}$}

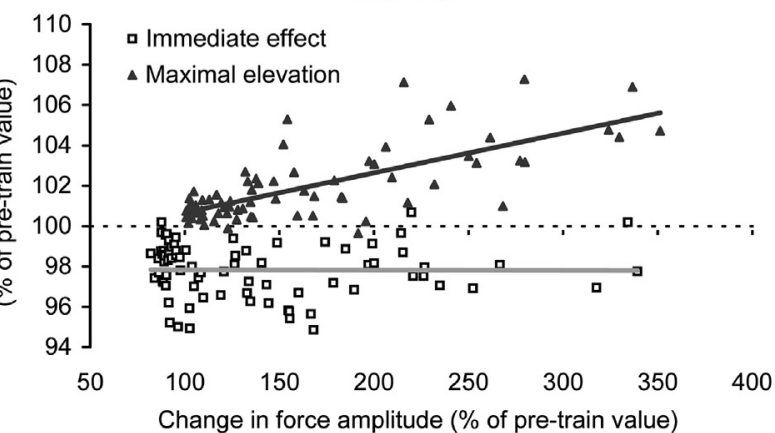

B

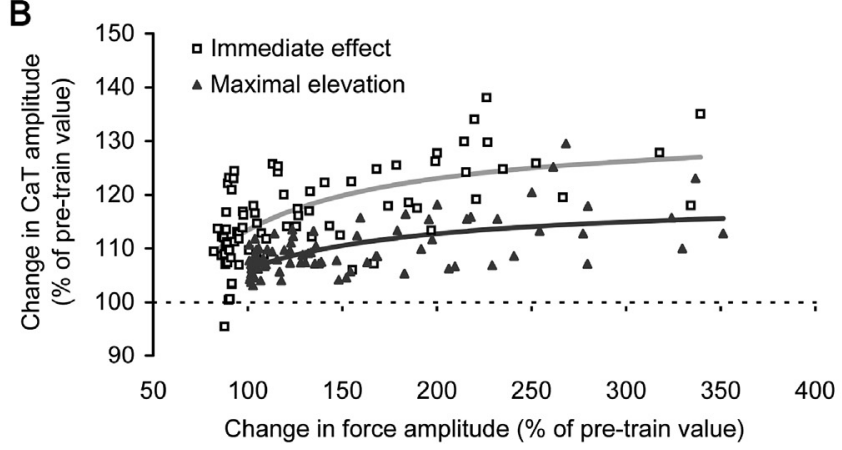

$1 \mathrm{~Hz}$
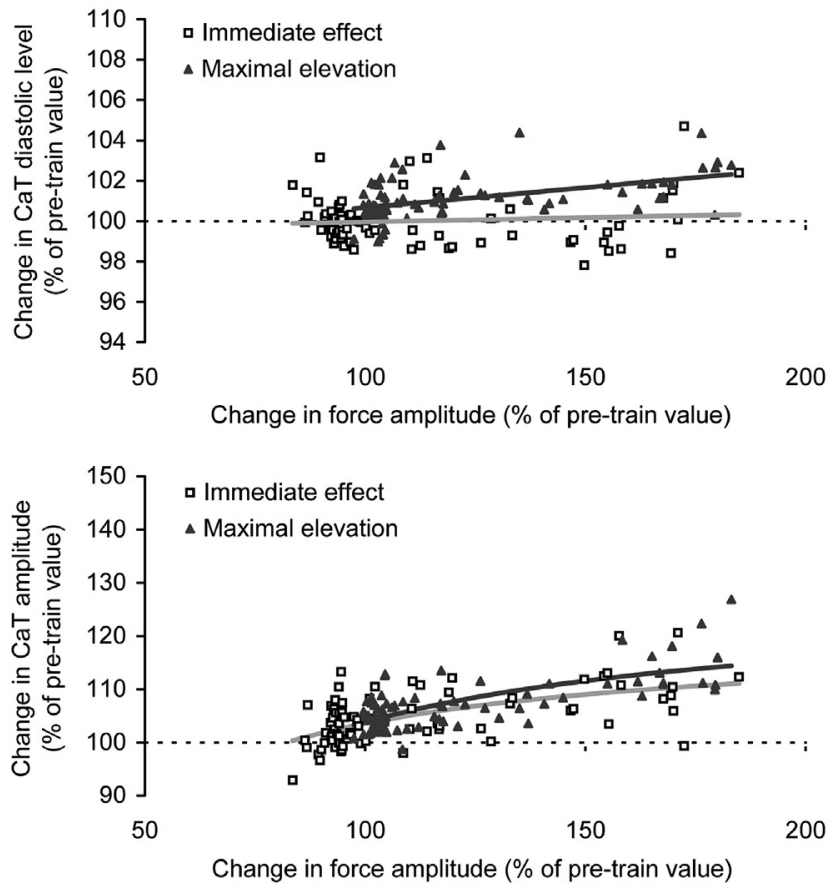

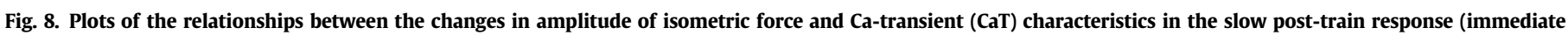

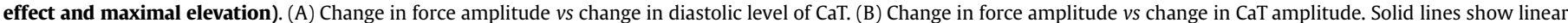

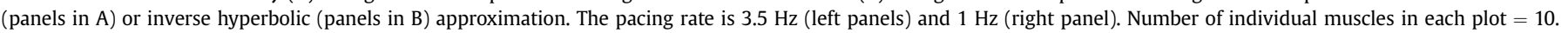

elevation of Ca-transient amplitude in the first post-train isometric twitch at the $1-\mathrm{Hz}$ pacing rate was independent of train length. In contrast, the $\downarrow-\uparrow$ trains at the $3.5-\mathrm{Hz}$ pacing rate produced significant and gradual train length dependent elevation of Catransient amplitude in the first post-train twitch. For the longest train ( $N=720$ cycles), the Ca-transient amplitudes in the first posttrain isometric twitch were $104.6 \pm 6.8 \%$ and $126.7 \pm 10.8 \%$ of the pre-train value at $1-\mathrm{Hz}$ and $3.5-\mathrm{Hz}$ pacing rates, respectively $(P<0.05)$. Thus, the highest effect of the $\downarrow-\uparrow$ train on the Catransient amplitude at the $3.5-\mathrm{Hz}$ pacing rate occurred in the first 
A

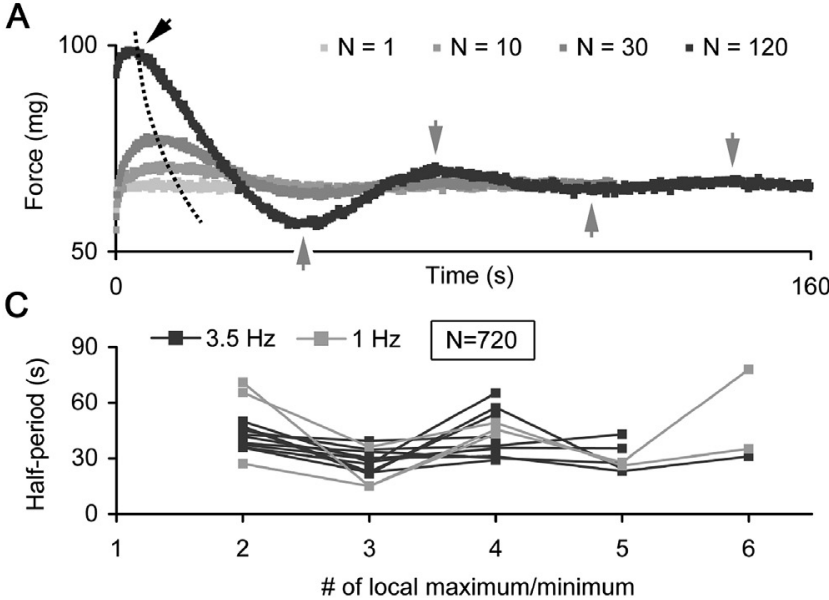

B
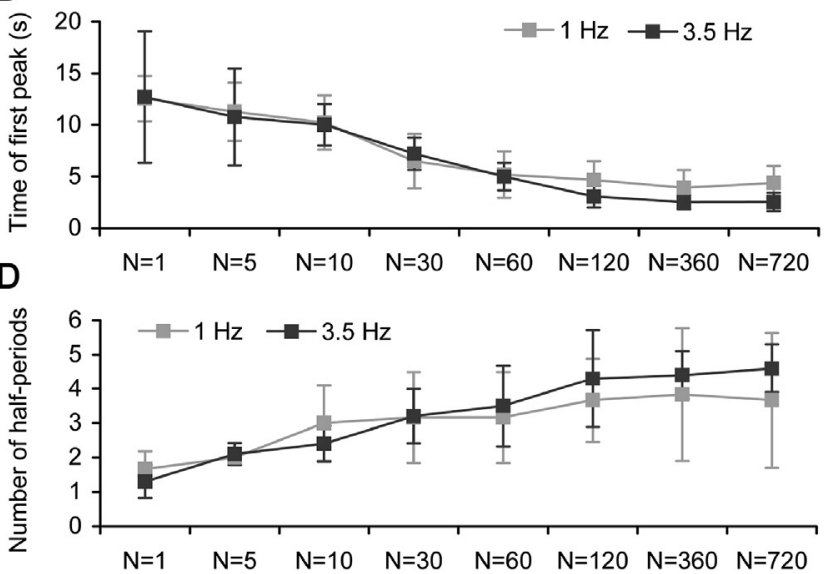

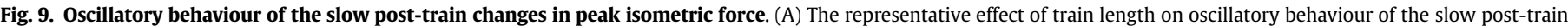

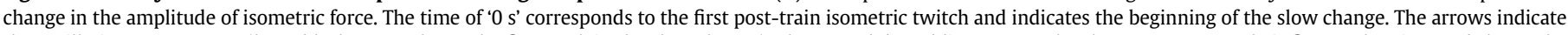

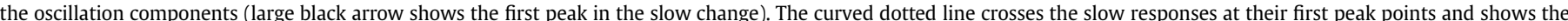

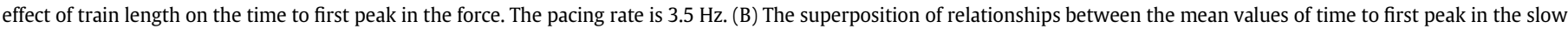

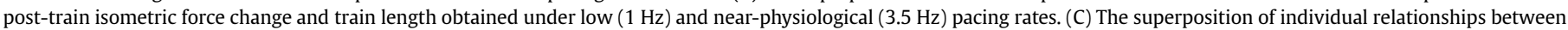

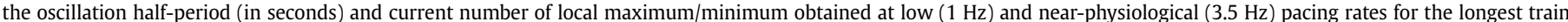

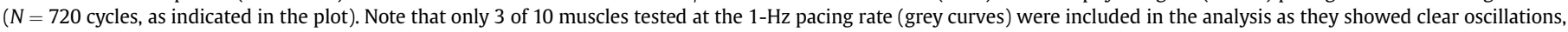

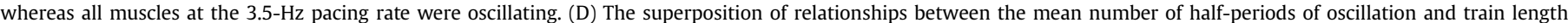

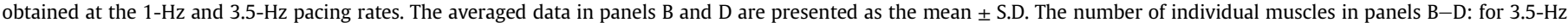
pacing rate, 10 in all panels; for $1-\mathrm{Hz}$ pacing rate, 6 in panels $\mathrm{B}$ and $\mathrm{D}, 3$ in panel C.

twitch because the maximal elevation during the slow post-train changes was only $\sim 116 \%$ of the pre-train value (compare Fig. $6 \mathrm{D}$ and $\mathrm{E}$ ). At the $1-\mathrm{Hz}$ pacing rate, the train produced similar magnitude of maximal elevation of Ca-transient amplitude in the slow post-train changes to that found for the $3.5-\mathrm{Hz}$ pacing rate (Fig. $6 \mathrm{E}$ ). The effect of the $\downarrow-\uparrow$ train on the minimal values of the Catransient diastolic level and amplitude in the slow post-train changes was not significant at the $1-\mathrm{Hz}$ pacing rate for any tested train length (Fig. $6 \mathrm{C}$ and $\mathrm{F}$ ). At the $3.5-\mathrm{Hz}$ pacing rate, a significantly higher effect of moderate train length on the Ca-transient diastolic level was found compared with the $1-\mathrm{Hz}$ pacing rate (Fig. 6C); however, no such difference was found for the Ca-transient amplitude (Fig. 6F).

The effects of train length on Ca-transient time-to-peak and time to decline to $50 \%$ amplitude $\left(T_{50}\right)$ were dissimilar from those obtained for the timing characteristics of the post-train isometric twitches. The immediate effect of the $\downarrow-\uparrow$ train on the Ca-transient time-to-peak value was negligible at any train length, irrespective of the pacing rate (Fig. 7A). Nevertheless, the delayed changes in the Ca-transient time-to-peak values contained both transient elevation and decrease during the slow post-train change (Fig. 7B and C, respectively). For example, the maximal elevations of the $\mathrm{Ca}$ transient time-to-peak values amounted to $150.5 \pm 25.4 \%$ and $146.3 \pm 23.7 \%$ of the pre-train values at the $1-\mathrm{Hz}$ and $3.5-\mathrm{Hz}$ pacing rates, respectively (no significant difference). Surprisingly, the maximal elevation and maximal decrease in the Ca-transient timeto-peak value were not significantly affected by the train length and were not significantly different between the $1-\mathrm{Hz}$ and $3.5-\mathrm{Hz}$ pacing rates (Fig. 7B and C). Similar effects of the $\downarrow-\uparrow$ train at the $1-\mathrm{Hz}$ and $3.5-\mathrm{Hz}$ pacing rates were found for $T_{50}$ of Ca-transient (Fig. 7D-F). The immediate effects were significantly different between $1-\mathrm{Hz}$ and $3.5-\mathrm{Hz}$ pacing rates at long enough trains (Fig. 7D). The maximal elevation of $T_{50}$ during the slow post-train change was significantly higher at the $3.5-\mathrm{Hz}$ pacing rate compared with the 1$\mathrm{Hz}$ pacing rate only for short trains $(N=5$ and 10 , Fig. $7 \mathrm{E})$, while no pacing-related difference was found for the maximal post-train decrease of $T_{50}$ at any tested train length (Fig. 7F).

The slow changes in the Ca-transient kinetics were found to be 'desynchronised' from the slow changes in the force. The minimal value of the diastolic level of Ca-transient and maximal value of its amplitude were both observed much earlier compared with the twitch where the maximal isometric force developed. The Catransient diastolic level was then slowly increased over the pretrain level and returned to this level (see black triangle in Fig. 5A). However, the Ca-transient amplitude rapidly decreased to the pre-train level. These time-unmatched slow changes in isometric force and Ca-transient characteristics indicate that sustained mechano-induced perturbations of (intra)cellular calcium content may produce not only immediate but also delayed mechanical output.

We plotted the immediate and maximal effects of the $\downarrow-\uparrow$ train on the peak isometric force against the same effects on the Catransient diastolic level or Ca-transient amplitude (Fig. 8). There was no correlation between the peak force and Ca-transient diastolic level when the immediate effects were compared (Fig. 8A, open square symbols and light grey lines). In contrast, the extent of maximal post-train (i.e. delayed) elevation of peak force showed a roughly linear relationship against the maximal elevation of the Catransient diastolic level (Fig. 8A, solid triangle symbols and dark grey lines). It should be noted that the $\sim 3$-fold augmentation of peak force at the $3.5-\mathrm{Hz}$ pacing rate is accompanied by a change of only a few percentage points in the Ca-transient diastolic level (Fig. 8A, left panel). The same was observed at the $1-\mathrm{Hz}$ pacing rate, including the quantitatively similar effects (Fig. 8A, right panel).

The immediate and delayed changes in Ca-transient amplitude (expressed as a percentage of the pre-train value) were substantially higher compared with the corresponding changes in the diastolic level and may achieve $130 \%$ of the pre-train value at the 3.5-Hz pacing rate (Fig. 8B, left panel). The plot between the effects on peak force and Ca-transient amplitude was substantially nonlinear compared with the plot of force vs Ca-transient diastolic level. At the $3.5-\mathrm{Hz}$ pacing rate, the same level of immediate effect on the force amplitude requires higher Ca-transient amplitude (Fig. 8B, left panel). In contrast, at the $1-\mathrm{Hz}$ pacing rate, we found no discrepancy between the plots for immediate and maximal delayed effects (Fig. 8B, right panel), likely due to the longer diastolic 
interval, which allows for recovery of the $\mathrm{Ca}^{2+}$ diastolic level at the end of twitch to its pre-train level (see Fig. 8A, right panel, open square symbols and light grey line). It was also found that the maximal delayed effect on force amplitude is achieved at the same value as the maximal delayed effect obtained for the Ca-transient amplitude $(\sim 110-112 \%$ of the pre-train level, dark grey lines in Fig. $8 \mathrm{~B}$, left and right panels).

\subsection{The oscillatory behaviour of the post-train inotropic response}

An increase in train length always resulted in the prolongation of the slow post-train recovery of contractility to the steady-state pre-train level (Fig. 9A). Moreover, we observed oscillatory behaviour of the slow changes in force, especially for long trains at high pacing rate.

First, we distinguished between the first peak of slow change and all subsequent local maxima and minima of the slow change (as indicated by black and grey arrows in Fig. 9A, respectively). We found that the time of the first peak in the slow change of force was substantially decreased with train length (Fig. 9A, as shown by the curved dotted line). Surprisingly, no significant difference in the effect of train length was found between $3.5-\mathrm{Hz}$ and $1-\mathrm{Hz}$ pacing rates (Fig. 9B); for example, the time to first peak in the slow change in the force decreased from $12.7 \pm 6.4 \mathrm{~s}(N=1)$ to $2.5 \pm 0.9 \mathrm{~s}$ $(N=720)$ at $3.5-\mathrm{Hz}$ and from $12.5 \pm 2.2 \mathrm{~s}(N=1)$ to $4.4 \pm 1.6 \mathrm{~s}$ $(N=720)$ at $1-\mathrm{Hz}$ pacing rate (mean \pm S.D.).

Second, we evaluated the timing properties of the oscillations if they were found during the slow change in the force. All the muscles paced at $3.5 \mathrm{~Hz}(n=10)$ showed highly prominent oscillations. In contrast, some muscles paced at $1 \mathrm{~Hz}$ did not show oscillatory behaviour, some displayed just single oscillation and only 3 of 10 muscles developed several oscillations. To retrieve the periodicity, we carefully examined the slow change in the force and found the oscillations as local maxima/minima (as shown by grey arrows in Fig. 9A). The interval between two consecutive local extremes in amplitude of force, for example, between the maximum and minimum or vice versa, was interpreted as a half-period of oscillatory behaviour. There was no substantial discrepancy between the half-period values for local extremes, measured in individual muscles at the $1-\mathrm{Hz}$ and $3.5-\mathrm{Hz}$ pacing rates and plotted against the sequence number of the extreme (Fig. 9C). This finding indicates that the slow change in isometric force triggered by the $\downarrow-\uparrow$ train at either pacing rate progresses with a nearly constant time factor (the half-period was $\sim 40 \mathrm{~s}$ for all tested muscles) and fading amplitude. The data shown in Fig. 9C were aggregated for the longest train ( $N=720$ cycles) because the most prominent oscillatory behaviour was observed for this train. However, it was found that the mean number of half-periods, which reflects the prominence of oscillation behaviour, was increased with the train length (Fig. 9D) from $1.3 \pm 0.5(N=1)$ to $4.6 \pm 0.7(N=720)$ at 3.5$\mathrm{Hz}$ pacing rate and from $1.7 \pm 0.5(N=1)$ to $3.7 \pm 2.0(N=720)$ at $1-$ $\mathrm{Hz}$ pacing rate (mean \pm S.D., no significant difference between the corresponding mean values at the $1-\mathrm{Hz}$ and $3.5-\mathrm{Hz}$ pacing rates).

Despite the prominent slow post-train oscillation of the isometric force, we did not find such behaviour for any of the Catransient characteristics (amplitude, time-to-peak, $T_{50}$ ). Possibly, we failed to observe such effects due to the much noisier fluorescent signals compared with the contractile responses of the muscle. On the other hand, as noted above, very fine changes in Catransient may induce great changes in contractility.

\section{Discussion}

The aims of our study were as follows: i) to evaluate the changes in myocardial inotropic response and Ca-transient kinetics after sustained twitch-by-twitch shortening-re-lengthening cycles, ii) to reveal the role of pacing rate in the extent of these changes, and iii) to infer the (intra)cellular mechanisms responsible for these effects.

\subsection{The modulation of $\mathrm{Ca}^{2+}$ homeostasis in cardiac muscle by shortening-re-lengthening trains}

According to the results of our study, the following explanation of shortening-re-lengthening modulation of $\mathrm{Ca}^{2+}$ homeostasis can be proposed. The shortening-induced partial inactivation of myofilaments, primarily via a length-dependent decrease in the $\mathrm{Ca}^{2+}$ sensitivity of troponin C (TnC) (ter Keurs, 2012; Biesiadecki et al., 2014), results in the elevation of non-utilised $\mathrm{Ca}^{2+}$ in the cytosol. This 'free' $\mathrm{Ca}^{2+}$ can partially leave the cell via the sodium-calcium exchanger (NCX) and plasma-membrane $\mathrm{Ca}^{2+}$-ATP pump (PMCA) (Louch et al., 2012). However, it is reasonable that most of the $\mathrm{Ca}^{2+}$ is pumped back to the sarcoplasmic reticulum (SR) because the shortening-induced decrease in $\mathrm{Ca}^{2+}$-buffering by TnC augments the buffering capacity of the SR (Briston et al., 2014). This increases the $\mathrm{Ca}^{2+}$ load of the SR and may also decrease the $\mathrm{Ca}^{2+}$ diastolic level to below the pre-train level, as demonstrated by the in-train Ca-transient in Fig. 5B (middle panel). The shifts in $\mathrm{Ca}^{2+}$ content may be minor in a single shortening-re-lengthening cycle, but can accumulate during a long train of such cycles (e.g. see Fig. 6D). On the other hand, the immediate and maximal delayed inotropic responses 'saturate' at long enough trains (Fig. 2A and B), which may indicate a limited capacity of the SR to accumulate $\mathrm{Ca}^{2+}$ ions and/or enhanced $\mathrm{Ca}^{2+}$ leakage from the SR (Shannon et al., 2002) and out of the cell via NCX (Trafford et al., 1998). The short train (<10 cycles) results in a negative immediate inotropic effect, and the moderate train (15-20 cycles) gives no immediate inotropy (see inset in Fig. 2D). This may indicate that the $\mathrm{Ca}^{2+}$ intake by the SR is not instantaneous, and the cumulative elevation of the SR $\mathrm{Ca}^{2+}$ load needs enough time to be achieved.

It was found that the substantial slow post-train inotropic changes are accompanied by less prominent alterations in the $\mathrm{Ca}-$ transient characteristics (Figs. 6-8). However, the relatively small changes in Ca-transients may affect numerous fast and deferred $\mathrm{Ca}^{2+}$-dependent processes, including phosphorylation of phospholamban and intracellular kinases (Gattoni et al., 2016), SERCA2a conformational shifts (Raguimova et al., 2018), regulation of nucleus Ca-transient (Kiess and Kockskämper, 2019) and posttranslational changes in $\mathrm{Ca}^{2+}$-regulating components (Biesiadecki et al., 2014; Smith and Eisner, 2019). Importantly, the changes in the $\mathrm{Ca}^{2+}$ diastolic level alone may induce positive inotropism (Shutt et al., 2006; Louch et al., 2012). In addition, the 'extra' repletion of the SR by non-utilised cytosolic $\mathrm{Ca}^{2+}$ may preserve the contractile function under myocardial diseases, similar to the compensatory elevation in SERCA2a expression in the right ventricle after left ventricular dysfunction following myocardial infarction (Fernandes et al., 2015). Such functional remodelling may facilitate $\mathrm{Ca}^{2+}$ loading of the SR during substantial shortening under a low afterload, which in turn, may provide 'extra' $\mathrm{Ca}^{2+}$ for positive inotropism after a sudden increase in aortic pressure. In contrast, impaired $\mathrm{Ca}^{2+}$ uptake by the SR in the failing myocardium decreases the systolic function under rapid changes in the afterload (Zima et al., 2014). Therefore, there is potential clinical significance in the development of strategies addressed to the improvement of impaired Ca handling and homeostasis (Kho et al., 2012).

The cardiac contraction and relaxation is determined not only by the kinetics of $\mathrm{Ca}^{2+}$ interaction with intracellular buffers (ter Keurs, 2012; Eisner et al., 2017) but also the kinetics of myosin crossbridges (Stehle and Iorga, 2010; Milani-Nejad et al., 2016; Reconditi et al., 2017). The inotropic response under physiological 
conditions depends on the level of peak systolic $\mathrm{Ca}^{2+}$, as well as the $\mathrm{Ca}^{2+}$ sensitivity of thin filament and its interaction with thick filament (Biesiadecki et al., 2014; Reconditi et al., 2017; Zhang et al., 2017). It is known that regulation of active tension at submaximal $\left[\mathrm{Ca}^{2+}\right]_{\mathrm{i}}$ strongly depends on the preceding sarcomere length (ter Keurs, 2012). Moreover, re-lengthening of sarcomeres in diastole additionally activates myofilaments due to the increased $\mathrm{Ca}^{2+}$ sensitivity of TnC and force-dependent recruitment of myosin cross-bridges (Farman et al., 2011; Milani-Nejad et al., 2016; Reconditi et al., 2017; Zhang et al., 2017; Campbell et al., 2018), and this plays a key role in the fast decrease of intraventricular pressure and early diastolic filling of ventricles (Stehle and Iorga, 2010). The number of cross-bridges remaining to be bound at diastole also affects the level of diastolic $\mathrm{Ca}^{2+}$ (Ferreira-Martins and LeiteMoreira, 2010), as well as the $\mathrm{Ca}^{2+}$ activation level in the following twitches (Smith and Eisner, 2019). In our mechanical tests, the shortening-re-lengthening was switched to isometric contraction in the diastole, and this may account for the additional length-dependent activation of force in the first isometric twitch. Indeed, the $\mathrm{Ca}^{2+}$ demand by sarcomeric myofilaments in the isometric state is increased due to the length-dependence of $\mathrm{Ca}^{2+}$ sensitivity of thin filaments (de Tombe et al., 2010; Ait-Mou et al., 2016; Zhang et al., 2017; Li et al., 2019) or by additional recruitment of myosin cross-bridges (Milani-Nejad et al., 2016; Reconditi et al., 2017; Campbell et al., 2018), and this demand can be satisfied by the accumulated $\mathrm{Ca}^{2+}$, as evidenced by the elevated Ca-transient amplitude (Fig. 6D). This agrees with findings that the dynamical change in sarcomere length, not the length per se, is important in the regulation of myocardial contractility (Tangney et al., 2014; Kobirumaki-Shimozawa et al., 2016). The slow inotropic response may reflect the combined effects of increased $\mathrm{Ca}^{2+}$-buffering by $\mathrm{TnC}$ via length-induced increase in $\mathrm{Ca}^{2+}$ sensitivity and tensionassociated effects on thin filaments (Dobesh et al., 2002; Dupuis et al., 2018; Li et al., 2019) and slow equilibration of $\mathrm{Ca}^{2+}$ content between the main $\mathrm{Ca}^{2+}$ buffers (Trafford et al., 2001; Louch et al., 2012).

\subsection{The role of the pacing rate in the mechano-induced immediate and delayed inotropic effects}

The inotropic response to changes in the stimulation rate is one of the key adaptive responses in the mammalian myocardium (Endoh, 2004; Janssen and Periasamy, 2007). An increase in the pacing rate elevates the L-type $\mathrm{Ca}^{2+}$ current and provides the compensatory augmentation of $\mathrm{Ca}^{2+}$ loading of the SR, giving a higher amount of $\mathrm{Ca}^{2+}$ release in the next twitch (Endoh, 2004; Janssen and Periasamy, 2007; Paterek et al., 2016). With time, the integral $\mathrm{Ca}^{2+}$ efflux through NCX and PMCA adjusts to compensate for altered $\mathrm{Ca}^{2+}$ influx through L-type channels; following this, the balance of $\mathrm{Ca}^{2+}$ influx and efflux is achieved at the new level with no net $\mathrm{Ca}^{2+}$ movement across the membrane during the entire twitch (Eisner et al., 2017).

While the SR rapidly releases $\mathrm{Ca}^{2+}$ into the cytosol of the activated cell, the time is needed to refuel SR. Therefore, the retention of an adequate diastolic interval is ultimately important for a proper cardiac cycle, and this is governed by the mechanisms of acceleration of relaxation under an increased pacing rate (FerreiraMartins and Leite-Moreira, 2010; Louch et al., 2012; Biesiadecki et al., 2014; Paterek et al., 2016; Røe et al., 2019). As the pacing rate increases, both force development and relaxation phases are shortened in a highly proportional manner (Janssen, 2010). This facilitates normal systolic function in the succeeding twitches because the diastolic interval remains long enough for normal repletion of the SR by $\mathrm{Ca}^{2+}$. In contrast, disproportional retardation of relaxation results in inadequate diastolic filling and a decrease in stroke volume (Selby et al., 2011; Biesiadecki et al., 2014). Nevertheless, a shortened diastolic interval at a high pacing rate limits the ability of $\mathrm{Ca}^{2+}$ to recover to the initial levels in all intracellular sources (Louch et al., 2012). The cumulative shift in $\mathrm{Ca}^{2+}$ homeostasis explains why the immediate and delayed inotropic effects of the shortening-re-lengthening train are significantly higher at the near-physiological pacing rate compared with the low one (see Fig. 2A and B).

As noted above, the mechano-induced immediate and delayed inotropic effects are much more prominent compared with the effects on Ca-transient characteristics both at low and high pacing rates (Fig. 8). However, the discrepancy between immediate and delayed effects was substantial only at the $3.5-\mathrm{Hz}$ pacing rate (see left and right panels in Fig. 8A and B). The discrepancy between the immediate and maximal delayed effects on the force amplitude $v s$ Ca-transient amplitude at the near-physiological pacing rate (Fig. 8B, left panel) may relate to the initially low Ca-transient diastolic level in the first post-train isometric twitch (open square symbols and light grey curve in Fig. 8A, left panel), which signals the SR to produce elevated Ca-transient. In the succeeding twitches, the Ca-transient diastolic level gradually increases over the pretrain level (as indicated by black triangle in Fig. 5A), and this may signal the SR to release a smaller amount of $\mathrm{Ca}^{2+}$ (smaller Catransient amplitude). At the low pacing rate, a longer diastolic interval gives enough time for the diastolic level of $\mathrm{Ca}^{2+}$ to restore to the pre-train level (see Fig. $8 \mathrm{~A}$, right panel, open square symbols and light grey line). On the other hand, the maximal delayed inotropic effect at both the pacing rates corresponds to the same value as the maximal delayed effect on Ca-transient amplitude (compare dark grey lines in the left and right panels of Fig. 8B). This indicates that the amount of 'free' non-utilised $\mathrm{Ca}^{2+}$ is pumped back to the SR, and that this process does not depend on the pacing rate.

\subsection{Oscillatory behaviour of the mechano-induced delayed inotropic response}

In the present study, we observed the oscillatory behaviour of slow post-train inotropic response in all muscles paced at the nearphysiological rate and in some of these muscles paced at the low rate (see Fig. 9). It is unlikely that such oscillations are related to the well-known phenomenon of alternating contractions (pulsus alternans) in the mammalian myocardium (Eisner et al., 2000; Díaz et al., 2004). The alternant activity is triggered by the intermittent augmented and diminished $\mathrm{Ca}^{2+}$ releases from the SR under a certain imbalance between the SR $\mathrm{Ca}^{2+}$ content and peak cytosolic $\mathrm{Ca}^{2+}$; they appear to be more prominent under lowered peak systolic $\mathrm{Ca}^{2+}$ (Pogwizd and Bers, 2004; Fernandez-Tenorio and Niggli, 2018). In our experiments, the oscillations of force were larger at $3.5 \mathrm{~Hz}$ vs $1 \mathrm{~Hz}$ pacing rates and the Ca-transient amplitude was substantially lower at $3.5 \mathrm{~Hz} v s 1 \mathrm{~Hz}$ in each trabecula subjected to testing (data not shown). Nevertheless, the observed oscillations did not reveal a regular twitch-by-twitch change, which is typical of alternating twitches; instead, they developed during tens of twitches. We suggest that these oscillations may reflect the slowly fluctuating equilibration between two main intracellular $\mathrm{Ca}^{2+}$ buffers - the SR and thin myofilaments. The relatively stable duration of single oscillation under the low and near-physiological pacing rates in most of the muscles (see Fig. 9C) supports this hypothesis because it is unlikely to observe pacing-dependent alteration in inherent features of SERCA2a and TnC. Consequently, the total duration of the slow recovery of contractility may depend on two main factors: i) the cumulative disturbance of the $\mathrm{Ca}^{2+}$ balance induced by sustained mechanical perturbation and ii) the rates of the main $\mathrm{Ca}^{2+}$-utilising mechanisms contributing to the recovery of 
$\mathrm{Ca}^{2+}$ balance (SERCA2a, NCX, etc). Assuming the latter mechanisms are independent of the actual $\mathrm{Ca}^{2+}$ levels here and there (e.g. if NCX moves $\mathrm{Ca}^{2+}$ ions with a constant rate), enhanced accumulation of $\mathrm{Ca}^{2+}$ in the SR at a high pacing rate will result in a higher amplitude of oscillating response (darkest curve in Fig. 9A), but the number of oscillations and the total time needed to recover the $\mathrm{Ca}^{2+}$ content to the pre-perturbation levels will remain independent of the pacing rate (see Fig. 9D). Similarly, the result shown in Fig. 9D indicates that a longer shortening-re-lengthening train simply induces a higher extent of accumulated imbalance between (intra) cellular calcium sources; therefore, a longer time is needed to achieve the initial pre-train level of $\mathrm{Ca}^{2+}$ content in these sources.

\subsection{Does the shortening-re-lengthening-induced inotropism relate} to the other known mechano-induced inotropic responses?

The phenomenon of mechano-induced modulation of succeeding contractions has been demonstrated in our recent studies (Katsnelson et al., 2011; Markhasin et al., 2012; Balakin et al., 2018). It has been shown that regular systolic shortening of $\mathrm{Ca}^{2+}$-overloaded cardiac muscle may induce spontaneous $\mathrm{Ca}^{2+}$ release from the SR and out-of-rhythm contractions (Katsnelson et al., 2011). Moreover, the direction of length change, that is, preferential shortening or lengthening during a twitch, has a great effect on $\mathrm{Ca}^{2+}$ utilisation by myofilaments and $\mathrm{Ca}^{2+}$ uptake by the SR (Markhasin et al., 2012). The intra-myocardial slow force changes are physiologically important because the mechanical interaction of heterogeneous myocardial layers provides proper function to the whole heart (Cazorla and Lacampagne, 2011; Markhasin et al., 2012), whereas an impaired pattern of heterogeneity results in desynchronisation of the interaction and disturbs the normal diastolic function, filling and ejection of the ventricle (Ferreira-Martins and Leite-Moreira, 2010; Røe et al., 2019). The ability of mechanical perturbation to induce positive inotropism in the succeeding beats may play a role in the regulation of normal contractile function of the healthy heart and facilitate adaptive preservation of contractility in the diseased myocardium. We conclude that the mechanoinduced immediate and delayed effects, described in the present study, are of the same nature and potential significance as the intramyocardial slow force changes.

Another well-known phenomenon of the Slow Force Response (SFR), which occurs after a sudden stretching of cardiac muscle (for a review, see (Dowrick et al., 2019)), is related to distinct mechanisms despite the fact that they also result in the slow elevation of $\mathrm{Ca}^{2+}$ load in SR. (Only in this respect is SFR reminiscent of the slow inotropic responses shown in this study). We distinguish between them because of the principally different role of the mechanical condition in the initiation of these responses. Indeed, muscle length remains fixed after a sudden stretch and the $\mathrm{Ca}-\mathrm{TnC}$ kinetics plays a leading role in the signalling that may regulate intracellular $\mathrm{Ca}^{2+}$ content; it should be emphasised that the 'extra' $\mathrm{Ca}^{2+}$, which is accumulated in the SR, comes from the extracellular space. In contrast, sustained twitch-to-twitch shortening-re-lengthening of the muscle during its active state makes $\mathrm{Ca}^{2+}$-buffering by TnC less important in the regulation of $\mathrm{Ca}^{2+}$ content; the elevated amount of non-utilised cytosolic $\mathrm{Ca}^{2+}$ ions induces enhanced $\mathrm{Ca}^{2+}$ sequestration into the SR. Moreover, it is likely that the 'extra' $\mathrm{Ca}^{2+}$ accumulated in the SR has an intracellular origin; that is, it is not supplied from the extracellular space. Here, a process of redistribution of initially available $\mathrm{Ca}^{2+}$ between (intra)cellular sources is triggered to achieve a new $\mathrm{Ca}^{2+}$ balance at the given mechanical conditions. As the mechanical perturbation suddenly ceases, the accumulated $\mathrm{Ca}^{2+}$ is redistributed back to the pre-perturbation balance.

\section{Conclusion}

The immediate and delayed inotropic responses, which occurred after the sustained shortening-re-lengthening train in this study, reflect the combined effect of several mechano-sensitive mechanisms of regulation of myocardial contractility. The molecular basis of the regulation represents effective feedback between the $\mathrm{Ca}^{2+}$ influx, $\mathrm{Ca}^{2+}$ utilisation by intracellular buffers (mainly by the sarcoplasmic reticulum and regulatory protein troponin $C$ ) and $\mathrm{Ca}^{2+}$ efflux, which is modulated by the dynamical changes in cell length and/or load. The extent of the inotropic effects is strongly elevated under a near-physiological pacing rate due to the increased effect of a shortened diastolic interval on the $\mathrm{Ca}^{2+}$ balancing between intracellular sources. These mechano-induced positive inotropic responses may indicate the contractile reserve of the myocardium; that is, 'extra' $\mathrm{Ca}^{2+}$ is stored in the SR when the cardiac muscle works against a low load, but this $\mathrm{Ca}^{2+}$ is immediately supplied to the myofilaments when the muscle starts to work against a high load. Our findings elucidate a role of pacingdependent mechano-induced modulation of contractility via mechano-calcium feedback in the healthy myocardium and open a prospect for further studies on how this phenomenon is expressed and its importance in a diseased myocardium with substantially impaired $\mathrm{Ca}^{2+}$ homeostasis (e.g. heart failure).

\section{Author contributions}

OL, XB and YP contributed conception and design of the study. $\mathrm{OL}$ and $\mathrm{XB}$ performed experiments and data analysis. OL prepared first draft of the manuscript. All authors contributed to manuscript revision, read and approved the submitted version.

\section{Funding}

The study was supported by RFBR grant \#18-04-00572 and by RF Government Act \#211 of March 16, 2013 (agreement 02.A03.21.0006), and was carried out within the framework of the IIF UrB RAS theme No AAAA-A18-118020590031-8.

\section{Declaration of competing interest}

None.

\section{CRediT authorship contribution statement}

Oleg Lookin: Conceptualization, Methodology, Software, Formal analysis, Investigation, Writing - original draft, Writing review \& editing, Visualization, Funding acquisition. Xenia Butova: Methodology, Formal analysis, Investigation, Writing - review \& editing. Yuri Protsenko: Conceptualization, Methodology, Writing - review \& editing.

\section{Appendix A. Supplementary data}

Supplementary data to this article can be found online at https://doi.org/10.1016/j.pbiomolbio.2020.05.005.

\section{References}

Ait-Mou, Y., Hsua, K., Farman, G.P., Kumara, M., Greaser, M.L., Irving, T.C., de Tombe, P.P., 2016. Titin strain contributes to the Frank-Starling law of the heart by structural rearrangements of both thin- and thick-filament proteins. Proc. Natl. Acad. Sci. U.S.A. 113 (8), 2306-2311. https://doi.org/10.1073/ pnas.1516732113.

Balakin, A., Kuznetsov, D., Protsenko, Y., 2018. The phenomena of mechanical interaction of segments of hypertrophied myocardium. Prog. Biophys. Mol. Biol. 
133, 20-26. https://doi.org/10.1016/j.pbiomolbio.2017.10.002.

Biesiadecki, B.J., Davis, J.P., Ziolo, M.T., Janssen, P.M., 2014. Tri-modal regulation of cardiac muscle relaxation; intracellular calcium decline, thin filament deactivation, and cross-bridge cycling kinetics. Biophys. Rev. 6 (3-4), 273-289. https://doi.org/10.1007/s12551-014-0143-5.

Briston, S.J., Dibb, K.M., Solaro, R.J., Eisner, D.A., Trafford, A.W., 2014. Balanced changes in Ca buffering by SERCA and troponin contribute to $\mathrm{Ca}$ handling during $\beta$-adrenergic stimulation in cardiac myocytes. Cardiovasc. Res. 104 (2), 347-354. https://doi.org/10.1093/cvr/cvu201.

Campbell, K.S., Janssen, P.M.L., Campbell, S.G., 2018. Force-dependent recruitment from the myosin off state contributes to length-dependent activation. Biophys. J. 115 (3), 543-553. https://doi.org/10.1016/j.bpj.2018.07.006.

Cazorla, O., Lacampagne, A., 2011. Regional variation in myofilament lengthdependent activation. Pflügers Archiv 462 (1), 15-28. https://doi.org/10.1007/ s00424-011-0933-6.

de Tombe, P.P., Mateja, R.D., Tachampa, K., Ait Mou, Y., Farman, G.P., Irving, T.C. 2010. Myofilament length dependent activation. J. Mol. Cell. Cardiol. 48 (5), 851-858. https://doi.org/10.1016/j.yjmcc.2009.12.017.

Díaz, M.E., O’Neill, S.C., Eisner, D.A., 2004. Sarcoplasmic reticulum calcium content fluctuation is the key to cardiac alternans. Circ. Res. 94, 650-656. https:| doi.org/10.1161/01.RES.0000119923.64774.72.

Dobesh, D.P., Konhilas, J.P., de Tombe, P.P., 2002. Cooperative activation in cardiac muscle: impact of sarcomere length. Am. J. Physiol. Heart Circ. Physiol. 282 (3) H1055-H1062. https://doi.org/10.1152/ajpheart.00667.2001.

Dowrick, J.M., Tran, K., Loiselle, D.S., Nielsen, P.M.F., Taberner, A.J., Han, J.-C. Ward, M.-L., 2019. The slow force response to stretch: controversy and contradictions. Acta Physiol. 226 (1), e13250 https://doi.org/10.1111/apha.13250.

Dupuis, L.J., Lumens, J., Arts, T., Delhaas, T., 2018. High tension in sarcomeres hinders myocardial relaxation: a computational study. PloS One 13 (10), e0204642. https://doi.org/10.1371/journal.pone.0204642.

Eisner, D.A., Caldwell, J.L., Kistamás, K., Trafford, A.W., 2017. Calcium and excitationcontraction coupling in the heart. Circ. Res. 121, 181-195. https://doi.org/ 10.1161/CIRCRESAHA.117.310230.

Eisner, D.A. Choi, H.S., Díaz, M.E., O’Neill, S.C., Trafford, A.W., 2000. Integrative analysis of calcium cycling in cardiac muscle. Circ. Res. 87 (12), 1087-1094. https://doi.org/10.1161/01.res.87.12.1087.

Endoh, M., 2004. Force-frequency relationship in intact mammalian ventricula myocardium: physiological and pathophysiological relevance. Eur. J. Pharmacol. 500 (1-3), 73-86. https://doi.org/10.1016/j.ejphar.2004.07.013.

Farman, G.P., Gore, D., Allen, E., Schoenfelt, K., Irving, T.C., de Tombe, P.P., 2011 Myosin head orientation: a structural determinant for the Frank-Starling relationship. Am. J. Physiol. Heart Circ. Physiol. 300 (6), H2155-H2160. https:// doi.org/10.1152/ajpheart.01221.2010.

Fernandes, A.A., Ribeiro Jr., R.F., de Moura, V.G.C., Siman, F.D., Dias, F.M.V. Zoghaib, J., Vassallo, P.F., Vassallo, D.V., Stefanon, I., 2015. SERCA-2a is involved in the right ventricular function following myocardial infarction in rats. Life Sci. 124, 24-30. https://doi.org/10.1016/j.lfs.2015.01.010.

Fernandez-Tenorio, M., Niggli, E., 2018. Stabilization of Ca2+ signaling in cardiac muscle by stimulation of SERCA. J. Mol. Cell. Cardiol. 119, 87-95. https://doi.org 10.1016/j.yjmcc.2018.04.015.

Ferreira-Martins, J., Leite-Moreira, A.F., 2010. Physiologic basis and pathophysiologic implications of the diastolic properties of the cardiac muscle. J. Biomed. Biotechnol. 2010, 807084 https://doi.org/10.1155/2010/807084.

Gattoni, S., Røe, Å.T., Frisk, M., Louch, W.E., Niederer, S.A., Smith, N.P., 2016. The calcium-frequency response in the rat ventricular myocyte: an experimenta and modelling study. J. Physiol. 594 (15), 4193-4224. https://doi.org/10.1113/ JP272011.

Hanft, L.M., Korte, F.S., McDonald, K.S., 2008. Cardiac function and modulation of sarcomeric function by length. Cardiovasc. Res. 77 (4), 627-636. https://doi.org $10.1093 / \mathrm{cvr} / \mathrm{cvm} 099$

Janssen, P.M.L., 2010. Kinetics of cardiac muscle contraction and relaxation are linked and determined by properties of the cardiac sarcomere. Am. J. Physiol Heart Circ. Physiol. 299, H1092-H1099. https://doi.org/10.1152 ajpheart.00417.2010.

Janssen, P.M.L., Periasamy, M., 2007. Determinants of frequency-dependent contraction and relaxation of mammalian myocardium. J. Mol. Cell. Cardiol. 43 (5), 523-531. https://doi.org/10.1016/j.yjmcc.2007.08.012.

Katsnelson, L.B., Solovyova, O., Balakin, A., Lookin, O., Konovalov, P., Protsenko, Yu, Sulman, T. Markhasin, V.S., 2011. Contribution of mechanical factors to arrhythmogenesis in calcium overloaded cardiomyocytes: model predictions and experiments. Prog. Biophys. Mol. Biol. 107, 81-89. https://doi.org/10.1016 j.pbiomolbio.2011.06.001.

Kho, C., Lee, A., Hajjar, R.J., 2012. Altered sarcoplasmic reticulum calcium cycling targets for heart failure therapy. Nat. Rev. Cardiol. 9 (12), 717-733. https:// doi.org/10.1038/nrcardio.2012.145.

Kiess, T., Kockskämper, J., 2019. SERCA activity controls the systolic calcium increase in the nucleus of cardiac myocytes. Front. Physiol. 10, 56. https://doi.org/ 10.3389/fphys.2019.00056.

Kobirumaki-Shimozawa, F., Oyama, K., Shimozawa, T., Mizuno, A., Ohki, T., Terui, T. Minamisawa, S., Ishiwata, S., Fukuda, N., 2016. Nano-imaging of the beating mouse heart in vivo: importance of sarcomere dynamics, as opposed to sarcomere length per se, in the regulation of cardiac function. J. Gen. Physiol. 147 (1), 53-62. https://doi.org/10.1085/jgp.201511484.
Korte, F.S., Feest, E.R., Razumova, M.V., Tu, A.Y., Regnier, M., 2012. Enhanced Ca2+ binding of cardiac troponin reduces sarcomere length dependence of contractile activation independently of strong crossbridges. Am. J. Physiol. Heart Circ. Physiol. 303 (7), H863-H870. https://doi.org/10.1152/ajpheart.00395.2012.

Li, K., Methawasin, M., Tanner, B.C.W., Granzier, H.L., Solaro, R.J., Dong, W.-J., 2019. Sarcomere length-dependent effects on Ca2+-troponin regulation in myocardium expressing compliant titin. J. Gen. Physiol. 151 (1), 30-41. https://doi.org/ $10.1085 / j g p .201812218$.

Louch, W.E., Stokke, M.K., Sjaastad, I., Christensen, G., Sejersted, O.M., 2012. No rest for the weary: diastolic calcium homeostasis in the normal and failing myocardium. Physiology 27, 308-323. https://doi.org/10.1152/ physiol.00021.2012.

Markhasin, V.S., Balakin, A., Katsnelson, L.B., Konovalov, P., Lookin, O., Protsenko, Yu, Solovyova, O., 2012. Slow force response and auto-regulation of contractility in heterogeneous myocardium. Prog. Biophys. Mol. Biol. 110, 305-318. https:/| doi.org/10.1016/j.pbiomolbio.2012.08.011.

Milani-Nejad, N., Brunello, L., Gyorke, S., Janssen, P.M.L., 2014. Decrease in sarcoplasmic reticulum calcium content, not myofilament function, contributes to muscle twitch force decline in isolated cardiac trabeculae. J. Muscle Res. Cell Motil. 35 (3-4), 225-234. https://doi.org/10.1007/s10974-014-9386-9.

Milani-Nejad, N., Chung, J.-H., Canan, B.D., Davis, J.P., Fedorov, V.V., Higgins, R.S.D., Kilic, A., Mohler, P.J., Janssen, P.M.L., 2016. Insights into length-dependent regulation of cardiac cross-bridge cycling kinetics in human myocardium. Arch. Biochem. Biophys. 601, 48-55. https://doi.org/10.1016/j.abb.2016.02.005.

Paterek, A., Kępska, M., Kołodziejczyk, J., Maczewski, M., Mackiewicz, U., 2016. The effect of pacing frequency on the amplitude and time-course of cardiomyocyte shortening in isolated rat cardiomyocytes. Post. N. Med. XXIX (12B), 9-14. https://doi.org/10.5604/08606196.1226986.

Pogwizd, S.M., Bers, D.M., 2004. Cellular basis of triggered arrhythmias in heart failure. Trends Cardiovasc. Med. 14, 61-66. https://doi.org/10.1016/ j.tcm.2003.12.002.

Raguimova, O.N., Smolin, N., Bovo, E., Bhayani, S., Autry, J.M., Zima, A.V., Robia, S.L., 2018. Redistribution of SERCA calcium pump conformers during intracellular calcium signaling. J. Biol. Chem. 293 (28), 10843-10856. https://doi.org/10.1074/ jbc.RA118.002472.

Reconditi, M., Caremania, M., Pinzautia, F., Powersa, J.D., Narayananc, T., Stienend, G.J.M., Linaria, M., Lombardia, V., Piazzesi, G., 2017. Myosin filament activation in the heart is tuned to the mechanical task. Proc. Natl. Acad. Sci. Unit. States Am. 114 (12), 3240-3245. https://doi.org/10.1073/pnas.1619484114.

Røe, Å.T., Ruud, M., Espe, E.K., Manfra, O., Longobardi, S., Aronsen, J.M., Norden, E.S. Husebye, T. Kolstad, T.R.S., Cataliotti, A., Christensen, G., Sejersted, O.M. Niederer, S.A., Andersen, G.Ø., Sjaastad, I., Louch, W.E., 2019. Regional diastolic dysfunction in post-infarction heart failure: role of local mechanical load and SERCA expression. Cardiovasc. Res. 115, 752-764. https://doi.org/10.1093/cvr/ cvy257.

Selby, D.E., Palmer, B.M., LeWinter, M.M., Meyer, M., 2011. Tachycardia-induced diastolic dysfunction and resting tone in myocardium from patients with a normal ejection fraction. J. Am. Coll. Cardiol. 58, 147-154. https://doi.org/ 10.1016/j.jacc.2010.10.069.

Shannon, T.R., Ginsburg, K.S., Bers, D.M., 2002. Quantitative assessment of the SR Ca2+ leak-load relationship. Circ. Res. 91 (7), 594-600. https://doi.org/10.1161/ 01.RES.0000036914.12686.28.

Shutt, R.H., Ferrier, G.R., Howlett, S.E., 2006. Increases in diastolic [Ca2+] can contribute to positive inotropy in Guinea pig ventricular myocytes in the absence of changes in amplitudes of $\mathrm{Ca} 2+$ transients. Am. J. Physiol. Heart Circ Physiol. 291, H1623-H1634. https://doi.org/10.1152/ajpheart.01245.2005.

Smith, G.L., Eisner, D.A., 2019. Calcium buffering in the heart in health and disease.

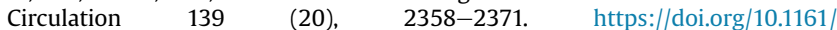
CIRCULATIONAHA.118.039329.

Stehle, R., Iorga, B., 2010. Kinetics of cardiac sarcomeric processes and rate-limiting steps in contraction and relaxation. J. Mol. Cell. Cardiol. 48 (5), 843-850. https://doi.org/10.1016/j.yimcc.2009.12.020.

Tangney, J.R., Campbell, S.G., McCulloch, A.D., Omens, J.H., 2014. Timing and magnitude of systolic stretch affect myofilament activation and mechanical work. Am. J. Physiol. Heart Circ. Physiol. 307, H353-H360. https://doi.org/ 10.1152/ajpheart.00233.2014.

ter Keurs, H.E.D.J., 2012. The interaction of $\mathrm{Ca} 2+$ with sarcomeric proteins: role in function and dysfunction of the heart. Am. J. Physiol. Heart Circ. Physiol. 302 (1), H38-H50. https://doi.org/10.1152/ajpheart.00219.2011.

Trafford, A.W., Diaz, M.E., Eisner, D.A., 1998. Stimulation of Ca-induced Ca release only transiently increases the systolic Ca transient: measurements of Ca fluxes and sarcoplasmic reticulum Ca. Cardiovasc. Res. 37, 710-717. https://doi.org/ 10.1016/s0008-6363(97)00266-6.

Trafford, A.W., Diaz, M.E., Eisner, D.A., 2001. Coordinated control of cell Ca2+ loading and triggered release from the sarcoplasmic reticulum underlies the rapid inotropic response to increased L-type Ca2+ current. Circ. Res. 88, 195-201. https://doi.org/10.1161/01.res.88.2.195.

Zhang, X., Kampourakis, T., Yan, Z., Sevrieva, I., Irving, M., Sun, Y.B., 2017. Distinct contributions of the thin and thick filaments to length-dependent activation in heart muscle. eLife 6, e24081. https://doi.org/10.7554/eLife.24081.

Zima, A.V., Bovo, E., Mazurek, S.R., Rochira, J.A., Li, W., Terentyev, D., 2014. Ca handling during excitation-contraction coupling in heart failure. Pflügers Archiv 466 (6), 1129-1137. https://doi.org/10.1007/s00424-014-1469-3. 